ESAIM: COCV 27 (2021) 82

https://doi.org/10.1051/cocv/2021064
ESAIM: Control, Optimisation and Calculus of Variations

www.esaim-cocv.org

\title{
A GEOMETRIC CONDITION FOR THE UNIFORM STABILITY OF LINEAR MAGNETOELASTICITY
}

\author{
ThOMAS DUYCKAERTS*
}

\begin{abstract}
We give a necessary and sufficient condition, of geometrical type, for the uniform decay of energy of solutions of the linear system of magnetoelasticity in a bounded domain with smooth boundary. A Dirichlet-type boundary condition is assumed. Our strategy is to use microlocal defect measures to show suitable observability inequalities on high-frequency solutions of the Lamé system.
\end{abstract}

Mathematics Subject Classification. 74F15, 35Q74, 35M10, 35L05.

Received November 15, 2020. Accepted June 12, 2021.

\section{INTRODUCTION}

Let $\Omega$ be a bounded, simply connected domain of $\mathbb{R}^{3}$, with a smooth boundary. Let us consider the following system, modelling the displacement of an elastic solid in a magnetic field:

$$
\begin{aligned}
& \left.\begin{array}{c}
\partial_{t}^{2} v-\varsigma \Delta v-(\lambda+\varsigma) \nabla \operatorname{div} v-\kappa \operatorname{curl} h \wedge \boldsymbol{B}=0 \\
\beta \partial_{t} h+\operatorname{curl} \operatorname{curl} h-\beta \operatorname{curl}\left(\partial_{t} v \wedge \boldsymbol{B}\right)=0 \\
\operatorname{div} h=0
\end{array}\right\}(t, y) \in(0, \infty) \times \Omega \\
& v=0, \quad h \cdot \mathbf{n}=0, \quad \operatorname{curl} h \wedge \mathbf{n}=0 \quad(t, y) \in(0, \infty) \times \partial \Omega,
\end{aligned}
$$

where $v=\left(v_{1}, v_{2}, v_{3}\right)$ is the displacement vector of the solid, and $h=\left(h_{1}, h_{2}, h_{3}\right)$ the magnetic field. The system is located in a constant exterior magnetic field $\boldsymbol{B}=(B, 0,0)$. We have denoted by $\Delta, \nabla$, div, curl respectively the Laplace operator, gradient, divergence and curl operators according to the space variable $y$, in the Euclidean metric of $\mathbb{R}^{3}$. The positive constants $\kappa$ and $\beta$ are coupling constants, and $\mathbf{n}$ is the external normal vector to the boundary of $\Omega$. The real Lamé constants $\lambda$ and $\varsigma$ are such that $\lambda+2 \varsigma>0, \varsigma>0$ and $\lambda+\varsigma \neq 0$.

The system (1.1) has a natural energy:

$$
E(t)=\frac{1}{2} \int_{\Omega}\left|\partial_{t} v\right|^{2}+\varsigma|\nabla v|^{2}+(\lambda+\varsigma)|\operatorname{div} v|^{2}+\kappa|h|^{2} \mathrm{~d} y
$$

Keywords and phrases: Magnetoelasticity, uniform stability, microlocal defect measure.

LAGA (UMR 7539), Université Sorbonne Paris Nord, Villetaneuse, France, and Institut Universitaire de France.

* Corresponding author: duyckaer@math.univ-paris13.fr 
which is time-decreasing. More precisely:

$$
\forall t \geq 0, \quad E(t)-E(0)=-\frac{\kappa}{\beta} \int_{0}^{t} \int_{\Omega}|\operatorname{curl} h(s, y)|^{2} \mathrm{~d} y \mathrm{~d} s .
$$

When $\Omega$ is simply connected, G. Perla Menzala and E. Zuazua have shown that this energy tends to zero as time tends to infinity, which is a simple consequence, using LaSalle invariance principle, of the non-existence of stationary solution for (1.1).

The system (1.1) may be seen as a coupling between the Lamé system

$$
\partial_{t}^{2} u-\varsigma \Delta u-(\lambda+\varsigma) \nabla \operatorname{div} u=0
$$

with Dirichlet boundary conditions, which is a conservative system, and the following heat equation:

$$
\beta \partial_{t} g-\Delta g=0
$$

The energy decay is produced by this strongly dissipative equation. From the point of view of $v$, the dissipation is caused by the coupling term: $R(v):=\operatorname{curl}\left(\partial_{t} v \wedge \boldsymbol{B}\right)$.

In this paper, we are interested by the possible uniform decay of the energy:

$$
E(t) \leq f(t) E(0), \quad f(t) \underset{t \rightarrow+\infty}{\longrightarrow} 0
$$

where $f$ if independent of the initial condition. In this case it is easy to show, using the semi-group property of the equation (1.1), that $f$ may be taken as a negative exponential function.

We will give, with a technical hypothesis on $\Omega$, a necessary and sufficient condition on the geometry of the problem for (1.3) to hold. When this condition is not fulfilled, there exist rays on $\Omega$, named $\boldsymbol{B}$-resistant rays, along which the energy of some solutions of (1.1) concentrates, and the dissipative term $R(v)$ is very small. Indeed, when such a ray exists, there is a sequence of solutions of (1.1) concentrating on the ray and which is in first approximation parallel to $\boldsymbol{B}$.

Before giving more explicit results, let us mention some earlier works on related subjects. As it was already stated, the convergence to 0 for the energy of magnetoelasticity in a bounded, simply connected domain was shown by G. Perla Menzala and E. Zuazua in [20], but their method does not give any information on the rate of convergence. By energy methods, Muñoz Rivera and Racke [18], Muñoz Rivera and de Lima Santos [8] have shown the rate of convergence to be at least polynomial, in dimension 2 or 3 , but only for some precise types of domains. Andreou and Dassios [1] have examined the same system on the entire space $\mathbb{R}^{3}$, showing again polynomial decay for some initial conditions. In [5], Charão, Oliveira and Perla Menzala have studied the system of magnetoelasticity in dimension 3 with an additional (linear or semilinear) damping term, and proved exponential or polynomial decay depending on the nature of the decay. See also [6] for a study in unbounded domains. Ma, Rivera, Oquendo and Suárez have studied the system of magnetoelastic plate (analogous to (1.1), where the wave equation is replaced by a plate equation) in [16].

The linear system of thermoelasticity is better understood. In this system, the Lamé system is coupled with a scalar heat equation. The dissipation is caused by the longitudinal part of the Lamé equation (the curl-free part of $v$ ). G. Lebeau and E. Zuazua [15] and N. Burq and G. Lebeau [3] have given (under a spectral assumption) a necessary and sufficient condition on $\Omega$, of geometrical nature, for the uniform decay in dimension 2 or 3 . Namely, this decay is equivalent to the non-existence of rays, called "transversal polarization rays", carrying the transversal component of $v$ (the divergence-free component), which resists to the dissipation. In [15], Lebeau and Zuazua have also proved the polynomial decay in dimension 2, under the same spectral assumption, which is namely that the operator associated to the equation does not admit any real eigenvalue. As shown in [20], this spectral condition is always fulfilled for the system of magnetoelasticity in a bounded, simply-connected domain. 
The comparison of the two systems of thermo and magnetoelasticity show that thermoelasticity is slightly less dissipative (the coupling of the Lamé system with the heat equation is weaker), and more difficult to describe, because of the non-trivial polarization of transversal waves.

Let us also mention the work [14] about the controllability of the system of thermoelasticity, and the articles [19], and [7] on the stability of the system of magneto-thermo-elasticity that take into accounts both thermic and magnetic effects.

The system of thermoelasticity of type III, where the classical heat equation is replaced by an hyperbolic (Cattaneo-type) heat equation was studied by Quintanilla and Racke [21] and Zhang and Zuazua in [24]. In this last article, the authors proved polynomial decay for most domain in dimension 2, and investigated uniform decay in dimension 3 , relying on the results of [15].

To state our main result, we need some geometrical preliminaries. Assume that $\partial \Omega$ has no contact of infinite order with its tangents. Thus, the Hamiltonian flow of the symbol of a wave operator $\partial_{t}^{2}-c^{2} \Delta$, which is defined locally in $S^{*}(\mathbb{R} \times \Omega)$ (the spherical cotangent bundle of $\Omega$ ), may be extended to the boundary of this bundle to a global flow, the generalized bicharacteristic flow, which may be seen as a continuous flow on the spherical compressed cotangent bundle $S_{b}^{*}(\mathbb{R} \times \bar{\Omega})(c f$. [11], Chap. 24.3). We shall call bicharacteristic rays or just rays the characteristic curves of this flow. Such a curve $\gamma$ will be said parallel to $\boldsymbol{B}$ if its direction of propagation is always parallel to $\boldsymbol{B}$ and orthogonal to $\boldsymbol{B}$ if its direction of propagation is always orthogonal to $\boldsymbol{B}$. We refer to Section 3 for the exact definitions of $S_{b}^{*}(\mathbb{R} \times \bar{\Omega})$ and of the generalized bicharacteristic flow.

The Lamé system (1.2) may be written as the sum of two wave equations known as the longitudinal and transversal wave equations, of respective speed $c_{L}:=\sqrt{\lambda+2 \varsigma}$ and $c_{T}:=\sqrt{\varsigma}$ (cf. Sect. 3.5). The assumption $\lambda+\varsigma \neq 0$ is equivalent to $c_{L} \neq c_{T}$.

Definition 1.1. One calls longitudinal ray (respectively transversal ray) any bicharacteristic ray for the operator $\partial_{t}^{2}-c_{L}^{2} \Delta$ (respectively $\partial_{t}^{2}-c_{T}^{2} \Delta$ ). One calls $\boldsymbol{B}$-resistant ray any continuous application $\gamma$ from an open interval $I=\left(s_{0}, s_{n}\right)$ to $S_{b}^{*}(\mathbb{R} \times \bar{\Omega})$ such that there exists a finite number of real numbers $s_{0}<s_{1}<\cdots<s_{n}$ such that

- on $\left(s_{j-1}, s_{j}\right), j \in\{1, \ldots, n\}, \gamma$ is a longitudinal ray parallel to $\boldsymbol{B}$, or a transversal ray orthogonal to $\boldsymbol{B}$;

- if $j \in\{1, \ldots, n-1\}, \gamma\left(s_{j}\right)$ is an hyperbolic point for the longitudinal and transversal waves (cf. Sect. 3.1.7) and one of the following assertions is true:

$\circ(L \rightarrow T)$ case: $\gamma$ is a longitudinal ray on $] s_{j-1}, s_{j}[$, and a transversal ray on $] s_{j}, s_{j+1}[$;

$\circ(T \rightarrow L)$ case: $\gamma$ is a transversal ray on $] s_{j-1}, s_{j}[$, and a longitudinal ray on $] s_{j}, s_{j+1}[$.

Some $\boldsymbol{B}$-resistant rays are drawn in Figure 1. The rays of this figure are all planar, but this is not a general property of $\boldsymbol{B}$-resistant rays.

Near $s_{j}, 1 \leq j \leq n-1$, the continuity imposed by the definition of $\gamma$ gives a condition on the angles of incidence and refraction. In the case $(L \rightarrow T)$, if we denote by $\alpha_{L}$ the angle between the longitudinal incoming ray and the tangent to $\partial \Omega$ in the plane of incidence, and by $\beta_{T}$ the angle between the transversal outcoming ray and this tangent, we have $\tan \alpha_{L}=\frac{c_{T}}{c_{L}}$ and $\tan \beta_{T}=\frac{c_{L}}{c_{T}}$ (which implies $\left.\alpha_{L}+\beta_{T}=\pi / 2\right)$. In the case $(T \rightarrow L)$, and with similar notations, we have $\tan \alpha_{T}=\frac{c_{L}}{c_{T}}$ and $\tan \beta_{L}=\frac{c_{T}}{c_{L}}$.

Theorem 1.2. Let $\Omega$ be a bounded, simply connected domain of $\mathbb{R}^{3}$, with a smooth boundary, having no contact of infinite order with its tangents. The energy of the system of magnetoelasticity in $\Omega$ decays uniformly if and only if there exists an $\ell>0$ such that the length of every $\boldsymbol{B}$-resistant ray on $\Omega$ is lesser than $\ell$.

Remark 1.3. As it will be shown in the proof, at high frequency, the transversal rays carry the component of $v$ which is orthogonal to the direction of propagation, and the longitudinal rays the component of $v$ which is parallel to this direction. A $\boldsymbol{B}$-resistant ray, whose direction of propagation is orthogonal to $\boldsymbol{B}$ in the transversal case and parallel to $\boldsymbol{B}$ in the longitudinal case carries essentially the component of $v$ which is parallel to $\boldsymbol{B}$, thus cancelling the dissipative term $R(v):=\operatorname{curl}\left(\partial_{t} v \wedge \boldsymbol{B}\right)$. From this point of view, Theorem 1.2 is very natural. 


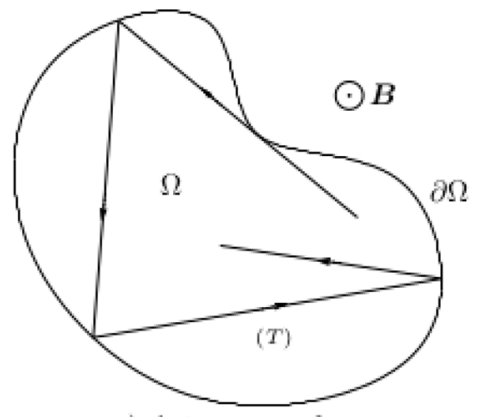

a) A transversal

$\boldsymbol{B}$-resistant ray

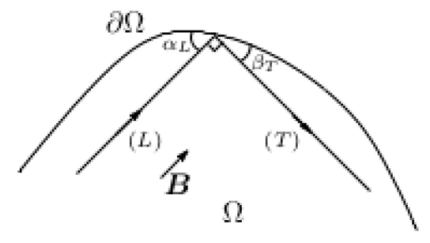

c) Transfer $(L \rightarrow T)$

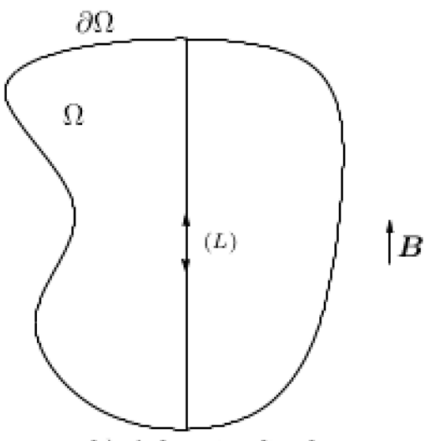

b) A longitudinal

$B$-resistant ray

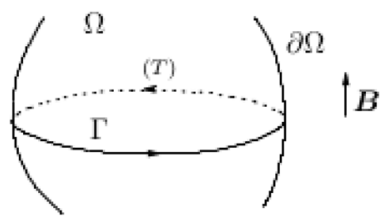

d) A boundary

$B$-resistant ray

Figure 1. Examples of $\boldsymbol{B}$-resistant rays.

Remark 1.4. It is essential to assume $c_{L} \neq c_{T}$. Otherwise, the first equation in (1.1) would be a wave equation with speed $c_{L}=c_{T}$. Every solution of (1.1) such that

$$
v_{\uparrow t=0} \perp \boldsymbol{B}, \quad \partial_{t} v_{\uparrow t=0} \perp \boldsymbol{B}, \quad h_{\uparrow t=0}=0
$$

would be of constant energy.

Remark 1.5. If $\Omega$ is not simply connected, there exists a finite dimensional space $E$ of stationary solutions of (1.1), whose components along $v$ are null. The study of the decay to zero of the solutions may be replaced by the study of their convergence to the eigenfunctions corresponding to the space $E$ ( $c f$. [20], Chap. 5). We won't develop this aspect here.

Remark 1.6. The condition of uniform decay is not fulfilled in simple cases, like the one of a ball, or an ellipsoid which has an axis parallel to the magnetic field. However it seems generic in the class of $C^{\infty}$ open sets. When this condition is not fulfilled, one can prove polynomial decay of regular solution (see the arXiv preprint [9]).

The proof uses propagation arguments on microlocal defect measures. This strategy of proof was initiated by G. Lebeau (see [12]).

The remainder of the paper is organized as follows. In Section 2, we reduce Theorem 1.2 to an high-frequency observability inequality on the Lamé system (1.2). This is based on two arguments: the setting aside of low frequencies, which is a consequence of the non-existence of stationary solution for the equation (1.1) shown in [20], and the decoupling, by simple calculations, of the two equations (the Lamé system and the heat equation) which compose (1.1). In Section 3, we introduce microlocal defect measures (an object due to P. Gérard [10] and L. Tatar [22], and in this particular setting to N. Burq and G. Lebeau [3]), in order to study the lack of compactness of a sequence of high-frequency solutions of the Lamé system. The main result of this section (apart from the existence of the measures), is a propagation theorem which was stated and shown in [3]. In Section 4, we prove the observability inequality on solutions of the Lamé system (1.2) which implies Theorem 1.2. The 
method of proof is to introduce, in a contradiction argument, a sequence of high frequency solutions of (1.2) which contradicts this inequality, and to use propagation arguments on the defect measures of this sequence. Section 5 is devoted to the necessary condition of Theorem 1.2, and is inspired by [4]: defect measures are used to construct a sequence of solutions of (1.2) concentrating on a $\boldsymbol{B}$-resistant ray and contradicting an observability inequality.

This paper is a corrected, updated and abbreviated ${ }^{1}$ version of the unpublished preprint [9] (2004), which is a translation of a chapter of the French PhD thesis of the author. The author would like to thank his former $\mathrm{PhD}$ supervisor, Nicolas Burq, for introducing him to this subject and more generally to microlocal analysis. This work is also indebted to Enrique Zuazua, who played an important role in the mathematical study of linear elasticity, especially with microlocal analysis techniques (see his articles with Gilles Lebeau [14] and [15]), and also has introduced system (1.1) in the mathematical community, in his work with G. Perla Menzala [20].

\section{OBSERVABILITY INEQUALITY FOR THE LAMÉ SYSTEM}

\subsection{Notations and preliminary results}

In this subsection are gathered a few basic facts about equations (1.1) and (1.2), as well as some notations. The main results of Section 2 are stated in the next subsection.

If $U$ is an open set of $\mathbb{R}^{3}$ or $\mathbb{R}^{4}$ we set

$$
\boldsymbol{H}^{s}(U):=H^{s}\left(U, \mathbb{C}^{3}\right), \quad \boldsymbol{L}^{2}(U):=L^{2}\left(U, \mathbb{C}^{3}\right)
$$

\subsubsection{Magnetoelasticity}

Consider the following spaces:

$$
\begin{aligned}
H:=\left\{g \in \boldsymbol{L}^{2}(\Omega), \operatorname{div} g\right. & =0 \text { in } \Omega, g \cdot n=0 \text { in } \partial \Omega\}, \quad \boldsymbol{H}_{0}^{1}:=\left\{f \in \boldsymbol{H}^{1}(\Omega), f=0 \text { in } \partial \Omega\right\} \\
H_{2} & :=\left\{f \in H \cap \boldsymbol{H}^{2}(\Omega), \operatorname{curl} f \wedge n=0 \text { in } \partial \Omega\right\},
\end{aligned}
$$

and the norms $\|g\|_{H}^{2}:=\kappa\|g\|_{\boldsymbol{L}^{2}(\Omega)}^{2},\|f\|_{\boldsymbol{H}_{0}^{1}(\Omega)}^{2}:=(\lambda+\varsigma)\|\operatorname{div} f\|_{L^{2}(\Omega)}^{2}+\varsigma\|\nabla f\|_{\left(\boldsymbol{L}^{2}(\Omega)\right)^{3}}^{2}$ Let $\mathcal{A}$ be the unbounded operator on $X:=\boldsymbol{H}_{0}^{1}(\Omega) \times \boldsymbol{L}^{2}(\Omega) \times H$, with domain $D(\mathcal{A})$, defined by

$$
\mathcal{A}\left(V_{0}\right):=\left(\begin{array}{c}
-v_{1} \\
-\Delta_{e} v_{0}-\kappa\left(\operatorname{curl} h_{0}\right) \wedge \boldsymbol{B} \\
-\operatorname{curl}\left(v_{1} \wedge \boldsymbol{B}\right)+\frac{1}{\beta} \operatorname{curl} \operatorname{curl} h_{0}
\end{array}\right), \quad D(\mathcal{A}):=\left(\boldsymbol{H}^{2} \cap \boldsymbol{H}_{0}^{1}\right) \times \boldsymbol{H}_{0}^{1} \times H_{2} .
$$

where $V_{0}=\left(v_{0}, v_{1}, h_{0}\right)$ denotes an element of $X$. Equation (1.1) may be rewritten

$$
\partial_{t} V+\mathcal{A} V=0, \quad V=\left(v, \partial_{t} v, h\right)
$$

The following proposition is due to G. Perla Menzala and E. Zuazua [20]:

Proposition 2.1. a) The operator $\mathcal{A}$ is maximal accretive. For any initial data $V_{0} \in X$, there exists a unique weak solution $V(t)=\left(v(t), \partial_{t} v(t), h(t)\right) \in C^{0}\left(\left[0,+\infty[; X)\right.\right.$ of $(2.1)$ such that $V(0)=V_{0}$.

b) The energy $E(t)$

$$
E(t):=\frac{1}{2}\|V(t)\|_{X}^{2}=\frac{1}{2} \int_{\Omega}\left|\partial_{t} v\right|^{2}+\varsigma|\nabla v|^{2}+(\lambda+\varsigma)|\operatorname{div} v|^{2}+\kappa|h|^{2} \mathrm{~d} y
$$

\footnotetext{
${ }^{\mathrm{I}} \mathrm{A}$ section on polynomial decay was taken off for the sake of brevity.
} 
is decreasing. More precisely,

$$
\forall t \geq 0, \quad E(t)-E(0)=-\frac{\kappa}{\beta} \int_{0}^{t} \int_{\Omega}|\operatorname{curl} h(s, y)|^{2} \mathrm{~d} y \mathrm{~d} s
$$

c) If $\Omega$ is simply connected, for all $V_{0}$ in $X, \lim _{t \rightarrow+\infty} E(t)=0$.

The assertions a) and b) are straightforward applications of the semi-group theory for the operator $\mathcal{A}$. The assertion c) is a consequence of the non-existence of stationary solutions for the system.

\subsubsection{Lamé system}

Let us now consider the Lamé system with Dirichlet boundary conditions:

$$
\begin{gathered}
\partial_{t}^{2} u-\Delta_{e} u=0 \text { in } \mathbb{R} \times \Omega \\
u_{\uparrow \partial \Omega}=0 \\
\left(u_{\uparrow t=0}, \partial_{t} u_{\uparrow t=0}\right)=\left(u_{0}, u_{1}\right) .
\end{gathered}
$$

Let $X_{e}$ be the space $\boldsymbol{H}_{0}^{1} \times \boldsymbol{L}^{2}$ and $\mathcal{L}$ the unbounded operator on $X_{e}$ defined by

$$
\mathcal{L}:=\left[\begin{array}{cc}
0 & -\mathrm{Id} \\
-\Delta_{e} & 0
\end{array}\right] \quad D(\mathcal{L}):=\left(\boldsymbol{H}^{2} \cap \boldsymbol{H}_{0}^{1}\right) \times \boldsymbol{L}^{2} .
$$

Taking $\left(u_{0}, u_{1}\right)$ in the energy space $X_{e}$, the equation (2.4) may be written

$$
\partial_{t} U+\mathcal{L} U=0, U(t)=\left(u, \partial_{t} u\right)
$$

Proposition 2.2. The operator $\mathcal{L}$ is maximal and unitary. For any initial data $U_{0}=\left(u_{0}, u_{1}\right) \in X_{e}$, the system (2.6) has a unique weak solution $U \in C^{0}\left(\mathbb{R}, X_{e}\right)$. Furthermore, the function $u$ is a solution of (2.4) in the distributional sense. At last, the energy

$$
\frac{1}{2}\left(\|u(t)\|_{\boldsymbol{H}_{0}^{1}}^{2}+\left\|\partial_{t} u\right\|_{\boldsymbol{L}^{2}}^{2}\right)=\frac{1}{2}\|U(t)\|_{X_{e}}^{2}
$$

of this solution is constant.

\subsubsection{Two useful lemma}

The two following standard lemma will be of great help in all this paper. The first one is due to the fact that $\Omega$ is simply connected ( $c f$. [23], Appendix I, Lem. 1.6):

Lemma 2.3. The $\boldsymbol{H}^{1}$ norm on $H \cap \boldsymbol{H}^{1}$ is equivalent to the norm $\|u\|:=\|\operatorname{curl} u\|_{L^{2}}$.

The second lemma is a elementary energy estimate on solutions of the non-homogeneous Lamé system. If $w(t)$ is a function with values in some Hilbert space, we set $\left(w_{0}, w_{1}\right):=\left(w, \partial_{t} w\right)_{\Gamma_{t=0}}$.

Lemma 2.4. Let $T>0, W \in C^{0}\left((0, T), X_{e}\right)$ and $F \in L^{2}\left((0, T), X_{e}\right)$ such that

$$
\partial_{t} W+\mathcal{L} W=F, \quad t \in(0, T)
$$


Then

$$
\int_{0}^{T}\|W(t)\|_{X_{e}}^{2} \mathrm{~d} t \leq C\left\{\|W(0)\|_{X_{e}}^{2}+\int_{0}^{T}\|F(t)\|_{X_{e}}^{2} \mathrm{~d} t\right\}
$$

where $C$ only depends on $T$. In particular, if

$$
\begin{aligned}
w \in C^{1}\left((0, T), \boldsymbol{L}^{2}(\Omega)\right) \cap & C^{0}\left((0, T), \boldsymbol{H}_{0}^{1}(\Omega)\right), \quad f \in \boldsymbol{L}^{2}((0, T) \times \Omega) \\
& \partial_{t}^{2} w-\Delta_{e} w=f,
\end{aligned}
$$

then

$$
\|w\|_{\boldsymbol{H}^{1}((0, T) \times \Omega)}^{2} \leq C\left(\left\|w_{0}\right\|_{\boldsymbol{H}^{1}(\Omega)}^{2}+\left\|w_{1}\right\|_{\boldsymbol{L}^{2}(\Omega)}^{2}+\|f\|_{\boldsymbol{L}^{2}((0, T) \times \Omega)}^{2}\right) .
$$

This is a standard energy inequality (using $\operatorname{Re}(\mathcal{L} W, W)_{X_{e}}=0$ ) and we omit the proof.

\subsection{Necessary and sufficient condition for uniform decay}

\subsubsection{Statement of the condition}

Proposition 2.5. Let $\Omega$ be a smooth, simply connected, bounded domain of $\mathbb{R}^{3}$.

a) Assume that there exist $T>0$ and $C>0$ such that for any solution $U$ of (2.6),

$$
\left\|u_{0}\right\|_{\boldsymbol{H}_{0}^{1}}^{2}+\left\|u_{1}\right\|_{\boldsymbol{L}^{2}}^{2} \leq C\left(\left\|\operatorname{curl}\left(\partial_{t} u \wedge \boldsymbol{B}\right)\right\|_{\boldsymbol{H}^{-1}((0, T) \times \Omega)}^{2}+\left\|u_{0}\right\|_{\boldsymbol{L}^{2}}^{2}+\left\|u_{1}\right\|_{\boldsymbol{H}^{-1}}^{2}\right) .
$$

Then the energy of solutions of the system of magnetoelasticity (1.1) decays uniformly.

b) Conversely, if the energy of solutions of (1.1) decays uniformly, then there exist $T>0$ and $C>0$ such that for any solution of (2.4) of finite energy,

$$
\left\|u_{0}\right\|_{\boldsymbol{H}_{0}^{2}}^{1}+\left\|u_{1}\right\|_{\boldsymbol{L}^{2}}^{2} \leq C\left\|\partial_{t} u(t) \wedge \boldsymbol{B}\right\|_{\boldsymbol{L}^{2}((0, T) \times \Omega)}^{2} .
$$

Remark 2.6. Proposition 2.5 is a fairly classical result in this setting. To prove it, we shall avoid the usual abstract decoupling argument (see [14]) but rather use simple energy estimates on the systems of magnetoelasticity and Lamé.

To prove Proposition 2.5, we first write a necessary and sufficient condition of uniform decay for solutions of a general dissipative equation. The second step of the proof consists in applying this condition to the system of magnetoelasticity, furthermore decoupling it in the system of Lamé and a heat equation.

\subsubsection{Abstract framework}

Let $\mathcal{P}$ be a maximal, accretive operator on an Hilbert space $X$, with dense domain $D(\mathcal{P})$. Denote by $\|.$.$\| the$ norm of $X,\|. .\|_{1}$ the natural norm on $D(\mathcal{P})$ and $\|. .\|_{-1}$ the norm on its dual space, with respect to the pivot space $X$. Assume that the embedding

$$
X \longrightarrow D(\mathcal{P})^{\prime}
$$


is compact. For $z_{0} \in X$, we will denote by $z(t)$ the solution (obtained for example by standard semi-group theory) of

$$
\frac{\mathrm{d} z}{\mathrm{~d} t}+\mathcal{P} z=0, \quad z_{t=0}=z_{0}
$$

By accretivity of $\mathcal{P}$, the energy $\frac{1}{2}\|z\|^{2}$ is time-decreasing. The following uniqueness-compactness argument is by now classical $(c f .[2])$ :

Lemma 2.7. The two following assertions (i) and (ii) are equivalent:

$$
\exists C>0, \exists a>0, \forall z_{0} \in X, \forall t>0,\|z(t)\|^{2} \leq C\left\|z_{0}\right\|^{2} e^{-a t}
$$

(the energy is uniformly decreasing)

a) $\exists T>0, \exists C>0, \forall z_{0} \in X,\|z(T)\|^{2} \leq C\left(\|z(0)\|^{2}-\|z(T)\|^{2}+\|z(0)\|_{-1}^{2}\right)$

b) There is no non-zero solution of (2.10) of constant energy on $[0,+\infty[$.

Corollary 2.8. The energy of (2.1) is uniformly time-decreasing if and only if

$$
\exists T>0, \exists C>0, \forall V_{0} \in X, E(T) \leq C\left\{\int_{0}^{T} \int_{\Omega}|\operatorname{curl} h|^{2} \mathrm{~d} y \mathrm{~d} t+\left\|V_{0}\right\|_{D(\mathcal{A})^{\prime}}^{2}\right\} .
$$

Indeed, the non-existence of stationary solution (the condition ii,b of Lemma 2.7 has been proved in [20], p. 356), which shows the corollary.

Proof of Lemma 2.7. It is easy to see that (i) may be replaced by

$$
\exists T>0, \exists C>0, \forall z_{0} \in X, \quad\|z(T)\|^{2} \leq C\left(\|z(0)\|^{2}-\|z(T)\|^{2}\right)
$$

Clearly (i') implies (ii).

Assume (ii). For some $T>0$, set

$$
q_{T}(z):=\|z(0)\|^{2}-\|z(T)\|^{2}, \quad G^{T}:=\left\{z_{0} \in X, q_{T}(z)=0\right\},
$$

which is the kernel of a positive, bounded, quadratic form on $X$, thus a closed subspace of $X$.

According to (ii), a) and the compactness of the embedding from $X$ to $D(P)^{\prime}, G(T)$ is locally compact, and thus of finite dimension, for large $T$. Combining with assumption b), we obtain that $G^{T}=\{0\}$ if $T$ is large enough.

Let us fix such a $T$. We prove $\left(i^{\prime}\right)$ by contradiction. Assume that there exists a sequence $\left(z_{0}^{k}\right)$ of elements of $X$ such that

$$
1=\left\|z^{k}(T)\right\|^{2}, \quad \lim _{k \rightarrow+\infty} q_{T}\left(z^{k}\right)=0 .
$$

Extracting subsequences, we may assume that $\left(z_{0}^{k}\right)$ converges weakly in $X$. Since $G^{T}=\{0\}$, its weak limit must be 0 . The compactness of the embedding of $X$ in $D(P)^{\prime}$ yields $\lim _{k \rightarrow+\infty}\left\|z_{0}^{k}\right\|_{-1}=0$, Contradicting $a$ ) and (2.11). 


\subsubsection{Proof of Proposition 2.5}

Assume the uniform time-decay of the energy of solutions of (2.1). Then, by (2.3), there exist $T>0$ and $C>0$ such that the following estimates hold for any solution $v$ of $(2.1)$

$$
\left\|v_{0}\right\|_{X}^{2} \leq C \int_{0}^{T}\|\operatorname{curl} h(t)\|_{L^{2}(\Omega)}^{2} \mathrm{~d} t
$$

Let $U$ be a solution of the Lamé system with initial data $U_{0}=\left(u_{0}, u_{1}\right) \in D(\mathcal{L})$ and $V$ the solution of the system of magnetoelasticity with initial data

$$
V_{0}=\left(v, \partial_{t} v, h\right)_{\lceil t=0}=\left(u_{0}, u_{1}, 0\right)
$$

Set: $W(t):=V(t)-\left(u(t), \partial_{t} u(t), 0\right)$. Then

$$
\partial_{t} W+\mathcal{A} W=\left(0,0,-\operatorname{curl}\left(\partial_{t} u \wedge \boldsymbol{B}\right)\right)
$$

Take the scalar product in $X$ with $W$ of the two sides of this equality, then integrate the real part with respect to time between 0 and $T$. Using

$$
\begin{gathered}
\operatorname{Re}(\mathcal{A} W, W)_{X}=\frac{\kappa}{\beta}\|\operatorname{curl} h\|_{\boldsymbol{L}^{2}(\Omega)}^{2} \\
\left(\operatorname{curl}\left(\partial_{t} u \wedge \boldsymbol{B}\right), h\right)_{\boldsymbol{L}^{2}(\Omega)}=\left(\partial_{t} u \wedge \boldsymbol{B}, \operatorname{curl} h\right)_{\boldsymbol{L}^{2}(\Omega)},
\end{gathered}
$$

the fact that $W_{\uparrow t=0}=0$, and in the second line, the inequality (2.12), we get

$$
\begin{gathered}
\|W(T)\|_{X}^{2}+\int_{0}^{T}\|\operatorname{curl} h(t)\|_{\boldsymbol{L}^{2}(\Omega)}^{2} \mathrm{~d} t \leq C \int_{0}^{T}\left\|\partial_{t} u(t) \wedge \boldsymbol{B}\right\|_{\boldsymbol{L}^{2}(\Omega)}^{2} \mathrm{~d} t \\
\left\|u_{0}\right\|_{\boldsymbol{H}_{0}^{1}(\Omega)}^{2}+\left\|u_{1}\right\|_{\boldsymbol{L}^{2}(\Omega)}^{2} \leq C \int_{0}^{T}\left\|\partial_{t} u(t) \wedge \boldsymbol{B}\right\|_{\boldsymbol{L}^{2}(\Omega)}^{2} \mathrm{~d} t
\end{gathered}
$$

This shows point b). To prove $a$ ), assume that inequality (2.8) holds. Consider a solution $V=\left(v, \partial_{t} v, h\right)$ of $(2.1)$ with initial data $V_{0}=\left(v_{0}, v_{1}, h_{0}\right)$, and the solution $u$ of the Lamé system with initial data

$$
\left(u, \partial_{t} u\right)_{\lceil t=0}=\left(v_{0}, v_{1}\right) .
$$

Thus, by (2.8):

$$
\left\|v_{0}\right\|_{\boldsymbol{H}_{0}^{1}}^{2}+\left\|v_{1}\right\|_{\boldsymbol{L}^{2}}^{2} \leq C\left(\left\|\operatorname{curl}\left(\partial_{t} u \wedge \boldsymbol{B}\right)\right\|_{\boldsymbol{H}^{-1}((0, T) \times \Omega)}^{2}+\left\|v_{0}\right\|_{\boldsymbol{L}^{2}}^{2}+\left\|v_{1}\right\|_{\boldsymbol{H}^{-1}}^{2}\right) .
$$

Furthermore, the energy inequality on the non-homogeneous Lamé system (Lem. 2.4) yields

$$
\begin{gathered}
\|u-v\|_{\boldsymbol{H}^{1}((0, T) \times \Omega)}^{2} \leq C \int_{0}^{T}\|\operatorname{curl} h(t)\|_{\boldsymbol{L}^{2}}^{2} \mathrm{~d} t \\
\left\|\operatorname{curl}\left(\partial_{t} u \wedge \boldsymbol{B}\right)\right\|_{\boldsymbol{H}^{-1}((0, T) \times \Omega)}^{2} \leq C\left\{\int_{0}^{T}\|\operatorname{curl} h(t)\|_{\boldsymbol{L}^{2}}^{2} \mathrm{~d} t+\left\|\operatorname{curl}\left(\partial_{t} v \wedge \boldsymbol{B}\right)\right\|_{\boldsymbol{H}^{-1}((0, T) \times \Omega)}^{2}\right\},
\end{gathered}
$$


which implies, using (2.13), Lemma 2.3 and the equation $\beta \partial_{t} h+\operatorname{curl} \operatorname{curl} h=\beta \operatorname{curl}\left(\partial_{t} v \wedge \boldsymbol{B}\right)$,

$$
\left\|v_{0}\right\|_{\boldsymbol{H}_{0}^{1}}^{2}+\left\|v_{1}\right\|_{\boldsymbol{L}^{2}}^{2} \leq C\left\{\int_{0}^{T}\|\operatorname{curl} h(t)\|_{\boldsymbol{L}^{2}}^{2} \mathrm{~d} t+\left\|v_{0}\right\|_{\boldsymbol{L}^{2}}^{2}+\left\|v_{1}\right\|_{\boldsymbol{H}^{-1}}^{2}\right\} .
$$

In order to use Corollary 2.8, we need to add to the left-hand side of inequality (2.14) the $\boldsymbol{L}^{2}$-norm of $h(T)$. We may do so by taking a larger $T$. Indeed, consider $s \in[0, T]$ such that

$$
\|h(s)\|_{\boldsymbol{L}^{2}}^{2}=\min _{t \in[0, T]}\|h(t)\|_{\boldsymbol{L}^{2}}^{2} .
$$

By Lemma 2.3,

$$
\|h(s)\|_{\boldsymbol{L}^{2}(\Omega)}^{2} \leq \frac{1}{T} \int_{0}^{T}\|h(t)\|_{\boldsymbol{L}^{2}(\Omega)}^{2} \mathrm{~d} t \leq \frac{C}{T} \int_{0}^{T}\|\operatorname{curl} h(t)\|_{\boldsymbol{L}^{2}(\Omega)}^{2} \mathrm{~d} t .
$$

Inequality (2.14) taken at the initial time $t=s$ yields

$$
E(s)=\frac{1}{2}\|V(s)\|_{X}^{2} \leq C\left(\int_{0}^{2 T}\|\operatorname{curl} h(t)\|_{L^{2}(\Omega)}^{2} \mathrm{~d} t+\|V(s)\|_{D(\mathcal{A})^{\prime}}^{2}\right) .
$$

Since $E$ and $\|V\|_{D(\mathcal{A})^{\prime}}$ are non-increasing functions of time, this implies the inequality of Corollary 2.8, and thus the uniform decay of solutions of (1.1). The proof of b) is complete.

\section{Defect Measures}

Let $N \geq 1$ be an integer (below we will take $N=1$ or $N=3$ ). For an open subset $U$ of an Euclidean space, we set

$$
\boldsymbol{L}^{2}(U):=L^{2}\left(U, \mathbb{C}^{N}\right), \quad \boldsymbol{H}^{s}(U):=H^{s}\left(U, \mathbb{C}^{N}\right)
$$

We consider an open subset $\Omega$ of $\mathbb{R}^{n}, n \geq 1$ (below we will take $n=3$ ), and a sequence $\left(u^{k}\right)$ of functions on $\mathbb{R}_{t} \times \Omega_{y}$ such that

$$
u_{k \rightarrow+\infty}^{k} 0 \text { in } \boldsymbol{H}_{\mathrm{loc}}^{1}(\mathbb{R} \times \bar{\Omega}),
$$

(in the sense that $\left(\varphi u^{k}\right)$ converges weakly to 0 in $\boldsymbol{H}^{1}(\mathbb{R} \times \bar{\Omega})$ for all $\varphi \in C_{0}^{\infty}(\mathbb{R} \times \bar{\Omega})$ ). We assume that every $u^{k}$ is solution of a wave equation in $\Omega$ :

$$
\left(\nu^{2} \partial_{t}^{2}-\Delta\right) u^{k}=0, \text { in } \mathbb{R} \times \Omega
$$

We shall introduce in this section a measure describing, from a microlocal point of view, the defect of compactness in $\boldsymbol{H}^{1}$ of the sequence $\left(u^{k}\right)$. This description is of fundamental importance to show the observability inequalities of the preceding section, since the Lamé system decomposes into two waves equation (see Sect. 3.5.1). Microlocal defect measures have been independently introduced by P. Gérard and L. Tatar [10, 22]. We shall follow the construction of N. Burq and G. Lebeau [3], which describes the defect of convergence up to the boundary of $\Omega$.

We assume, for the sake of simplicity that the functions $u^{k}$ are smooth, so that their traces on the boundary are always defined. In the sequel we shall always reduce to this case. 
In Section 3.1 we will give a few definitions and notations. In Section 3.2 we will state an existence theorem of microlocal defect measures and set out their first properties. Section 3.3 is devoted to the propagation theorem of the measure (proved in [3]), and Section 3.4 to some important properties of the traces of $u^{k}$ on the boundary. Finally, in Section 3.5, we shall apply the construction of the measure to the case of a sequence of solutions of the Lamé system.

\subsection{Notations}

\subsubsection{Local coordinates}

Consider an open cover of $\Omega: \Omega=\bigcup_{j=0}^{J} \Omega_{j}$, where $\bar{\Omega}_{0} \subset \Omega$ and, for all $j \geq 1, \Omega_{j}$ is a small neighbourhood of a point of $\partial \Omega$, such that on $\Omega_{j}$, there are geodesic normal coordinates

$$
\left.z \in \Omega_{j} \mapsto\left(y^{\prime}, x_{n}\right) \in Y:=Y^{\prime} \times\right] 0, l[
$$

where $x_{n}$ is the distance to the boundary, and $Y^{\prime}$ an open subset of $\mathbb{R}^{n-1}$. Most objects introduced here are global objects but we will mainly use local coordinates. For a large part of this section we choose one of the open set $\Omega_{j}, j \geq 1$.

Set $X:=\mathbb{R} \times Y, X^{\prime}:=\mathbb{R} \times Y^{\prime}$, and denote the elements of $X$ and $Y$ by

$$
x=(\underbrace{x_{0}, x_{1}, \ldots, x_{n-1}}_{x^{\prime}}, x_{n}), \quad x_{0}=t, \quad y=(\underbrace{x_{1}, x_{2}, \ldots, x_{n-1}}_{y^{\prime}}, x_{n}) \in Y,
$$

Let

$$
\mathbb{R}_{+}^{n+1}:=\left\{\left(x^{\prime}, x_{n}\right) \in \mathbb{R}^{n+1}, x_{n}>0\right\}, \quad \overline{\mathbb{R}}_{+}^{n+1}:=\overline{\mathbb{R}_{+}^{n+1}}, \quad \partial X=X^{\prime} \times\{0\}, \quad \bar{X}:=X^{\prime} \times[0, l[.
$$

The set $\bar{X}$ is an open subset of $\overline{\mathbb{R}}_{+}^{n}$. Let $g$ be the natural metric on $Y$, induced by the change of coordinates, and $g=\operatorname{det} g$. In a geodesic system of coordinates, $g$ is of the form

$$
g(y)=\left[\begin{array}{cc}
g^{\prime}(y) & 0 \\
0 & 1
\end{array}\right]
$$

\subsubsection{Bundles on $X$}

Let us consider $T^{*} X=X \times \mathbb{R}^{n+1}$ the cotangent bundle of $X$ and $S^{*} X$ the spherical cotangent bundle, which is defined to be the quotient

$$
S^{*} X:=\left(T^{*} X \backslash\{|\xi|=0\}\right) / \mathbb{R}_{+}^{*},
$$

by the action of $\mathbb{R}_{+}^{*}:(\lambda, \xi) \mapsto \lambda \xi$. The elements of those two bundles will be denoted by

$$
\rho=(x, \xi), \quad x \in X, \quad \xi=\left(\xi^{\prime}, \xi_{n}\right) \in \mathbb{R}^{n} \times \mathbb{R}, \quad \xi=(\tau, \eta)=\left(\tau, \eta^{\prime}, \xi_{n}\right) .
$$

There is a natural Euclidean norm for the $\eta$-component of $T^{*} X:\|\eta\|^{2}:={ }^{t} \eta g^{-1} \eta$.

We will also consider $T^{*} \bar{X}=\bar{X} \times \mathbb{R}^{n+1}, T^{*} \partial X:=\partial X_{x^{\prime}} \times \mathbb{R}_{\xi^{\prime}}^{n}$ the boundary cotangent bundle and $S^{*} \partial X$ the associated spherical bundle. 


\subsubsection{Operators in the interior of $\Omega$}

Le $S_{i}^{m}$ the set of matrix symbols of degree $m$ with compact support in $X$, which are the functions

$$
a(x, \xi) \in C^{\infty}\left(X \times \mathbb{R}^{n+1}, M_{N}(\mathbb{C})\right),
$$

such that there is a compact $K$ of $X$ with supp $a \subset K \times \mathbb{R}^{n+1}$, and satisfying the following estimates:

$$
\forall \alpha, \forall \beta, \exists C_{\alpha \beta},\left|\partial_{x}^{\alpha} \partial_{\xi}^{\beta} a(x, \xi)\right| \leq C_{\alpha \beta}(1+|\xi|)^{m-|\beta|},
$$

and which have a principal symbol $a_{m}(x, \xi)$, homogeneous function of degree $m$ in $\xi$, such that $a-a_{m}$ satisfies $\left(3.3_{m-1}\right)$ for large $|\xi|$. The operator of symbol $a, A=a(x, D)$, is defined by

$$
A v(x):=\frac{1}{(2 \pi)^{n+1}} \int a(x, \xi) \hat{v}(\xi) e^{i x \cdot \xi} \mathrm{d} \xi
$$

In order to act on functions which are only defined in $X$, it is convenient to consider only the set $\mathcal{A}_{i}^{m}$ consisting of operators $A$ which are of compact support in $X$, in the sense that $A=\varphi A \varphi$ for a function $\varphi \in C_{0}^{\infty}(X)$. An operator in $\mathcal{A}_{i}^{m}$ maps a distribution in $X$ to a compactly supported distribution in $X$. We shall denote by $\sigma_{m}(A)$ the principal symbol of an operator $A$ of degree $m$.

\subsubsection{Operators near the boundary}

Let $S_{b}^{m}$ be the set of tangential matrix symbols of degree $m$ with compact support in $X$, defined as the functions

$$
a\left(x, \xi^{\prime}\right) \in C^{\infty}\left(\bar{X} \times \mathbb{R}^{n}, M_{N}(\mathbb{C})\right)
$$

such that there is a compact $K$ of $\bar{X}$ with $\operatorname{supp} a \subset K \times \mathbb{R}^{n}$, and satisfying the estimates

$$
\forall \alpha, \forall \beta, \exists C_{\alpha \beta},\left|\partial_{x}^{\alpha} \partial_{\xi^{\prime}}^{\beta} a\left(x, \xi^{\prime}\right)\right| \leq C_{\alpha \beta}\left(1+\left|\xi^{\prime}\right|\right)^{m-|\beta|},
$$

and which have a principal symbol $a_{m}\left(x, \xi^{\prime}\right)$, homogeneous of degree $m$ in $\xi^{\prime}$ and such that $a-a_{m}$ satisfies the inequalities $\left(3.4_{m-1}\right)$ for large $\left|\xi^{\prime}\right|$. We define the operator of symbol $a, A=a\left(x, D^{\prime}\right)$, by

$$
A v(x):=\frac{1}{(2 \pi)^{n}} \int a\left(x, \xi^{\prime}\right) \hat{v}\left(\xi^{\prime}, x_{n}\right) e^{i x^{\prime} \cdot \xi^{\prime}} \mathrm{d} \xi^{\prime}
$$

Here, the Fourier transform of $v$ is only taken with respect to the tangential variable $x^{\prime}$. As in the interior case, we introduce the set $\mathcal{A}_{b}^{m}$ of tangential operators $A$ with compact support in $\bar{X}$, i.e. such that $A=\varphi A \varphi$ for a compactly supported function $\varphi \in C_{0}^{\infty}(\bar{X})$.

The set of all pseudo-differential operators of interest for us will be denoted by

$$
\mathcal{A}^{m}:=\left\{a=A_{i}+A_{b}, A_{i} \in \mathcal{A}_{i}^{m}, A_{b} \in \mathcal{A}_{b}^{m}\right\} .
$$

\subsubsection{Sobolev spaces}

Let $s \in \mathbb{R}$ and $\omega$ be an open set of $\mathbb{R}^{n}$ or $\mathbb{R}^{n+1}$. As before, we denote by $\boldsymbol{H}^{s}(\omega)$ the Sobolev space of $\mathbb{C}^{N}$ valued distributions (which is the set of restrictions to $\omega$ of elements of $\boldsymbol{H}^{s}\left(\mathbb{R}^{n}\right)$, endowed with the quotient 
norm). We also consider the space $\boldsymbol{H}_{\mathrm{loc}}^{s}(\omega)$, the space of vector-valued distributions such that

$$
\forall \varphi \in C_{0}^{\infty}(\omega), \quad \varphi u \in \boldsymbol{H}^{s}
$$

and $\boldsymbol{H}_{\text {comp }}^{s}(\omega)$, the space of distributions in $\boldsymbol{H}^{s}(\omega)$ compactly supported in $\omega$. The notation $\boldsymbol{H}_{\mathrm{loc}}^{s}(Z)$, will also be used when $Z$ is not open $(Z=\mathbb{R} \times \bar{\Omega}$, or $Z=\bar{X})$ as follows:

$$
u^{k} \underset{k \rightarrow+\infty}{\longrightarrow} u(\text { or }=O(1)) \text { in } \boldsymbol{H}_{\mathrm{loc}}^{s}(Z) \Longleftrightarrow \forall \varphi \in C_{0}^{\infty}(Z), \quad \varphi u_{k \rightarrow+\infty}^{k} \underset{\longrightarrow}{\longrightarrow} \varphi(=O(1)) \text { in } \boldsymbol{H}^{s}
$$

where $C_{0}^{\infty}(Z)$ is the space of $C^{\infty}$ functions whose support is a compact of $Z$. We will also consider the following spaces, suitable for boundary-value problems:

$$
\boldsymbol{H}_{\mathrm{loc}}^{0, s}(\bar{X})=L^{2}\left(0, l ; \boldsymbol{H}_{\mathrm{loc}}^{s}\left(X^{\prime}\right)\right), \quad \boldsymbol{H}_{\mathrm{comp}}^{0, s}(\bar{X})=\left\{u \in \boldsymbol{H}_{\mathrm{loc}}^{0, s}(\bar{X}), \exists \varphi \in C_{0}^{\infty}(\bar{X}), u=\varphi u\right\}
$$

The elements of $\mathcal{A}_{i}^{m}$ are continuous maps from $\boldsymbol{H}_{\text {loc }}^{s}(X)$ to $\boldsymbol{H}_{\text {comp }}^{s-m}(X)$, and those of $\mathcal{A}_{b}^{m}$ are continuous maps from $\boldsymbol{H}_{\mathrm{loc}}^{0, s}(\bar{X})$ to $\boldsymbol{H}_{\mathrm{comp}}^{0, s-m}(\bar{X})$.

Definition 3.1. Let $\rho \in S^{*} X$. The sequence $\left(v^{k}\right)$ is said to be bounded (respectively converging to 0 ) in $\boldsymbol{H}_{\rho}^{s}$ when there exists $A \in \mathcal{A}_{i}^{s}$, whose principal symbol is invertible near $\rho$ and such that $\left(A v^{k}\right)$ is bounded in $\boldsymbol{H}^{s}$ (respectively converges to 0 in $\boldsymbol{H}^{s}$ ).

Let $\rho^{\prime} \in S^{*} \partial X$. The sequence $\left(v^{k}\right)$ is said to be bounded (respectively converging to 0 ) in $\boldsymbol{H}_{\rho^{\prime}}^{0, s}$ when there exists $A \in \mathcal{A}_{b}^{s}$, whose principal symbol is invertible near $\rho^{\prime}$ and such that $\left(A v^{k}\right)$ is bounded in $\boldsymbol{H}^{0, s}$ (respectively converges to 0 in $\boldsymbol{H}^{0, s}$ ).

Note that, according to Proposition A.1 of the appendix, for a sequence of solutions of (3.2), the convergence in $\boldsymbol{H}^{0,1}$ and $\boldsymbol{H}^{1}$ are equivalent. The spaces $\boldsymbol{H}^{0,1}$ and the tangential operators are thus well fitted for the description of the $\boldsymbol{H}^{1}$ convergence of $\left(u^{k}\right)$.

\subsubsection{Melrose's compressed cotangent bundle}

We shall now introduce a bundle which naturally contains as subbundles both bundles $T^{*} X$ and $T^{*} \partial X$. For this purpose, set $T_{b}^{*} X:=\bar{X} \times \mathbb{R}^{n+1}$, endowed with its canonical topology and consider

$$
\begin{aligned}
T^{*} \bar{X} & \stackrel{j}{\longrightarrow} T_{b}^{*} X \\
\left(x, \xi^{\prime}, \xi_{n}\right) & \longmapsto\left(x, \xi^{\prime}, \theta=x_{n} \xi_{n}\right) .
\end{aligned}
$$

The mapping $j$ restricts to a continuous map:

$$
T^{*} X \longrightarrow T_{b}^{*} X \cap\left\{x_{n}>0\right\}
$$

which identifies $T^{*} X$ to a subbundle of dimension $2(n+1)$ of the interior of $T_{b}^{*} X$. Furthermore, the restriction of $j$ to $x_{n}=0$ defines a map from $T^{*} \bar{X} \cap\left\{x_{n}=0\right\}$ to $T_{b}^{*} X \cap\left\{x_{n}=0\right\}$, whose kernel is the set $\left\{\xi^{\prime}=0\right\}$. This clearly identifies

$$
T^{*} \partial X \approx\left(T^{*} \bar{X} \cap\left\{x_{n}=0\right\}\right) / \mathbb{R}_{\xi_{n}},
$$

(quotient taken by identifiying all the points $\left(\tilde{x}^{\prime}, \tilde{\xi}^{\prime}, \xi_{n}\right), \xi_{n} \in \mathbb{R}$ ) with a $2 n$-dimensional subbundle of $T_{b}^{*} X$. The set of all sections of $T_{b}^{*} X$, with the above identifications, may be seen as the dual bundle of the bundle of all vector fields on $X$ tangent to $\partial X$. It is called the compressed cotangent bundle. 
We will also consider $S_{b}^{*} X$ the spherical bundle of $T_{b}^{*} X$, which naturally contains the spherical bundles $S^{*} X$ and $S^{*} \partial X$.

\subsubsection{Symbol of $P$ and related manifolds}

The equation (3.2) takes the following form in local coordinates:

$$
P u^{k}=0, \quad P:=-\mathrm{g}^{-1 / 2} \partial_{x_{n}} \mathrm{~g}^{1 / 2} \partial_{x_{n}}+Q
$$

where $Q$ is a scalar tangential differential operator of degree 2. Let $q\left(x, \xi^{\prime}\right)$ be the principal symbol of $Q$, and $p(x, \xi)=\xi_{n}^{2}+q\left(x, \xi^{\prime}\right)$ the principal symbol of $P$. They are both scalar, homogeneous polynomials of degree 2 with respect to $\xi$. Let

$$
\text { Char } P:=\left\{(x, \xi) \in T^{*} \bar{X}, p(x, \xi)=0\right\}, \quad Z:=j(\operatorname{Char} P), \quad \widehat{Z}:=j(\operatorname{Char} P) \cup j\left(\left\{x_{n}=0\right\}\right),
$$

and $S$ Char $P, S Z, S \widehat{Z}$ the corresponding spherical bundles.

Denoting $q_{0}\left(x^{\prime}, \xi^{\prime}\right)=q\left(x^{\prime}, 0, \xi^{\prime}\right)$, we decompose $T^{*}(\partial X)$ (and $S^{*}(\partial X)$ ) into the disjoint union of the elliptic region $\mathcal{E}$, the glancing region $\mathcal{G}$ and the hyperbolic region $\mathcal{H}$ :

$$
\mathcal{E}:=\left\{q_{0}>0\right\}, \quad \mathcal{G}:=\left\{q_{0}=0\right\}, \quad \mathcal{H}:=\left\{q_{0}<0\right\}
$$

\subsubsection{Global measure}

The defect measure is at first constructed in each of the preceding local coordinate systems. The objects obtained are then pieced together to $M=\mathbb{R} \times \Omega$. It is easy to define from local objects global Sobolev spaces and bundles on $M$, such as Melrose's compressed cotangent bundle $T_{b}^{*} M$. We shall use the same notations (Char $P, Z, \widehat{Z}, S$ Char $P, S Z, S \widehat{Z}, \ldots$ ) for the local and global objects. The definition of global operators is less natural in our setting. The symbol $\mathcal{A}^{m}$ will denote the set of operators $A$ acting on functions on $M$, which are of the form

$$
A=\sum_{j=0}^{J} A_{(j)} .
$$

where $A_{(0)}$ is a classical pseudo-differential operator of order $m$ with compact support in $M$ and each $A_{(j)}$ is an operator of the sets $\mathcal{A}^{m}$ defined in each system of local coordinates. The global space $\mathcal{A}^{m}$ depends of the coordinate patches chosen, which shall not cause any problem in the remaining of the article. For a totally intrinsic construction, we could have used Melrose's totally characteristic operators (see [11], Chap. 18.3).

\subsection{Existence of the measure}

\subsubsection{The existence theorem}

The next elementary proposition ( $c f$. Lems. 2.3 and 2.4 of [3]) shows that for any $A \in \mathcal{A}^{0}$, the behaviour of $A u^{k}$ in $\boldsymbol{H}^{1}$ only depends upon the restriction of its principal symbol to $S \widehat{Z}$ :

Proposition 3.2. Let $A_{b} \in \mathcal{A}_{b}^{-\varepsilon}$. Let $\left(u^{k}\right)_{k}$ be as above, satisfying (3.1) and (3.2). Then

$$
A_{b} u^{k} \underset{k \rightarrow 0}{\longrightarrow} 0 \text { in } \boldsymbol{H}^{1}
$$


Let $A_{i} \in \mathcal{A}_{i}^{0}$, whose principal symbol vanishes on Char $P$. Then

$$
A_{i} u^{k} \underset{k \rightarrow 0}{\longrightarrow} 0 \text { in } \boldsymbol{H}^{1}
$$

According to Proposition 3.2, it is sufficient to describe the $\boldsymbol{H}^{1}$ convergence of $\left(u^{k}\right)$ near $S \widehat{Z}$, in the sense given by Definition 3.1. Let $\mathcal{M}$ be the set of matrix-valued measures on $S \widehat{Z}$, i.e. the dual space of

$$
\mathcal{C}:=C_{0}^{0}\left(S \widehat{Z}, M^{N}(\mathbb{C})\right)
$$

and $\mathcal{M}^{+}$the subset of all positive measures in $\mathcal{M}$, i.e. measures $\mu$ which satisfy

$$
\forall b \in \mathcal{C}, \quad(\forall z \in S \widehat{Z}, \quad b(z) \geq 0) \Longrightarrow\langle\mu, b\rangle \geq 0
$$

( $M \geq 0$ means $M$ is positive Hermitian).

Before coming to the main theorem of this paragraph, we shall introduce a technical condition on $u^{k}$ :

Definition 3.3. Let the sequence $\left(u^{k}\right)$ satisfies (3.1) and (3.2). We shall say that $\left(u^{k}\right)$ is regular on the boundary when one the following equivalent assumptions is satisfied:

$$
\begin{gathered}
u_{\uparrow x_{n}=0}^{k}=o(1) \text { in } \boldsymbol{H}_{\mathrm{loc}}^{1 / 2}(\partial X), k \longrightarrow+\infty \\
\partial_{x_{n}} u_{\uparrow x_{n}=0}^{k}=o(1) \text { in } \boldsymbol{H}_{\mathrm{loc}}^{-1 / 2}(\partial X), k \longrightarrow+\infty .
\end{gathered}
$$

Note that the standard trace theorems imply conditions (3.6) with $O(1)$ instead of $o(1)$. All the sequences $\left(u^{k}\right)$ in this work shall satisfy this condition. For the proof of the equivalence between (3.6a) and (3.6b) see Lemma 2.6 of [3].

Theorem 3.4. Let $u^{k}$ be such that (3.1), (3.2) and (3.6) hold. Then there exists a subsequence of $\left(u^{k}\right)$, still denoted by $\left(u^{k}\right)$, and a measure $\mu \in \mathcal{M}^{+}$, called microlocal defect measure of $\left(u^{k}\right)$, such that $\mu(\mathcal{E} \cup \mathcal{H})=0$ and

$$
\forall A_{j} \in \mathcal{A}^{j}, j \in\{1,2\}, \lim _{k \rightarrow+\infty}\left(A_{2} u^{k}+A_{1} D_{x_{n}} u^{k}, u^{k}\right)=\left\langle\mu, \frac{a_{2}+\xi_{n} a_{1}}{\tau^{2}}\right\rangle .
$$

In (3.7), the notation (.,.) stands for the $\boldsymbol{L}^{2}$-scalar product on $M$ (in local coordinates, it is the scalar product on $X$ using the metric $\left.\mathrm{g}^{1 / 2} \mathrm{~d} y \mathrm{~d} t\right)$. We note that $\tau \neq 0$ on $S \widehat{Z} \backslash(\mathcal{E} \cup \mathcal{H})$, and that

$$
\forall(x, \xi) \in S \widehat{Z} \cap\left\{x_{n}>0\right\}, \quad\left|\frac{\xi_{n} a_{1}}{\tau^{2}}\right| \leq \frac{\sqrt{q\left(x, \xi^{\prime}\right)}\left|a_{1}\left(x, \xi^{\prime}\right)\right|}{\tau^{2}},
$$

so that the function $(x, \xi) \mapsto \frac{\xi_{n} a_{1}}{\tau^{2}}$ extends to a continuous function on $S \widehat{Z} \backslash(\mathcal{E} \cup \mathcal{H})$, which is null on $\mathcal{G}$. Thus the right-hand side of (3.7) makes sense.

Remark 3.5. The measure $\tilde{\mu}=\mu \mathbb{1}_{\left\{x_{n}>0\right\}}$ may be seen as the standard microlocal defect measure (cf. [10]) of the bounded sequence $\left(u^{k}\right)$ of $\boldsymbol{H}_{\mathrm{loc}}^{1}(M)$. This interior measure describe the compactness defect of $\left(u^{k}\right)$ in $\boldsymbol{H}_{\mathrm{loc}}^{1}(M)$ (in particular, it is null when $\left(u^{k}\right)$ converges to 0 in this space), but not in $\boldsymbol{H}_{\mathrm{loc}}^{1}(\bar{M}): \tilde{\mu}$ vanishes when $\left(u^{k}\right)$ concentrates on $\partial M$, even if it does not converge to 0 in $\boldsymbol{H}_{\mathrm{loc}}^{1}(\bar{M})$. On the other hand,

$$
\varphi \in C_{0}^{\infty}(\bar{M}) \Longrightarrow \int \varphi\left|\nabla_{y} u^{k}\right|^{2} \mathrm{~d} x+\int \varphi\left|\partial_{t} u^{k}\right|^{2} \mathrm{~d} x \underset{k \rightarrow+\infty}{\longrightarrow}\langle\mu, \varphi\rangle
$$


The measure $\mu$ thus gives a complete description of the local convergence of $\left(u^{k}\right)$ in $\boldsymbol{H}^{1}(\bar{M})$. This description is indeed microlocal:

$$
\rho \in \operatorname{supp} \mu \Longleftrightarrow u^{k} \underset{k \rightarrow+\infty}{\longrightarrow} 0 \text { in } \begin{cases}\boldsymbol{H}_{\rho}^{1} & \text { if } x_{n}>0 \\ \boldsymbol{H}_{\rho}^{0,1} & \text { if } x_{n}=0 .\end{cases}
$$

Theorem 3.4 is a new formulation, using Lemma 2.7 of [3], of Proposition 2.5 of this article. Compared to this proposition, the measure $\mu$ of Theorem 3.4 has been renormalized.

\subsubsection{A sufficient condition of nullity for $\mu$}

Let $\tilde{\rho} \in S \widehat{Z}$ an interior point and $A \in \mathcal{A}_{i}^{2}$, whose principal symbol is invertible at $\tilde{\rho}$. By elementary symbolic calculus on classical operators, it is easy to show, with formula (3.7),

$$
A u^{k} \underset{k \rightarrow+\infty}{\longrightarrow} 0 \text { in } \boldsymbol{H}_{\tilde{\rho}}^{-1} \Longrightarrow \tilde{\rho} \notin \operatorname{supp} \mu \text {. }
$$

The same statement holds in $\mathcal{G}$ :

Proposition 3.6. Let $\tilde{\rho} \in \mathcal{G}$. Consider an operator of the form

$$
A=A_{0} D_{x_{n}}^{2}+A_{1} D_{x_{n}}+A_{2}, A_{j} \in \mathcal{A}^{j}, a_{j}:=\sigma\left(A_{j}\right),
$$

such that

$$
A u^{k} \underset{k \rightarrow+\infty}{\longrightarrow} 0 \text { in } \boldsymbol{H}_{\tilde{\rho}}^{0,-1}
$$

Assume that $\left(u^{k}\right)$ is regular on the boundary and that $a_{2}(\tilde{\rho})$ is invertible. Then

$$
\tilde{\rho} \notin \operatorname{supp} \mu \text {. }
$$

Remark 3.7. Proposition 3.6 is trivial when $A=A_{2} \in \mathcal{A}^{2}$ (it is essentially the definition of $\boldsymbol{H}_{\tilde{\rho}}^{0,-1}$ ).

Remark 3.8. Note that according to the appendix, the convergence to 0 of $\left(A u^{k}\right)$ in the space $\boldsymbol{H}^{-1}(X)$ near $\tilde{x}$ would imply (3.9). Furthermore, the proof of Proposition 3.6 will show that assumption (3.9) is equivalent to

$$
\left(B A u^{k}, u^{k}\right) \underset{k \rightarrow+\infty}{\longrightarrow} 0, \forall B \in \mathcal{A}^{0} \text {, with support close enough to } \tilde{\rho} \text {. }
$$

Proof. According to (3.8), $\mu \mathbb{1}_{\left\{x_{n}>0\right\}}$ is null near $\tilde{\rho}$. The same property remains to be proved on $\mu \mathbb{1}_{\left\{x_{n}=0\right\}}$. Let $B \in \mathcal{A}^{0}, \psi \in C_{0}^{\infty}(\mathbb{R})$ such that $\psi(0)=1, B_{\varepsilon}:=\psi\left(\frac{x_{n}}{\varepsilon}\right) B$, and $b=\sigma(B)$. In view of (3.9) and (3.7),

$$
\left\langle\mu, \psi\left(\frac{x_{n}}{\varepsilon}\right) b \frac{a_{0} \xi_{n}^{2}+a_{1} \xi_{n}+a_{2}}{\tau^{2}}\right\rangle=\lim _{k \rightarrow+\infty}\left(B_{\varepsilon} A u^{k}, u^{k}\right)=0
$$

Letting $\varepsilon$ goes to 0 , the dominated convergence theorem and the fact that $\xi_{n}$ is null on the support of $\mu \mathbb{1}_{\left\{x_{n}=0\right\}}$ give

$$
\left\langle\mu, \mathbb{1}_{\mathcal{G}} \frac{b a_{2}}{\tau^{2}}\right\rangle=0
$$


Let $\phi \in S_{b}^{0}$ be scalar, positive, and compactly supported near $\tilde{\rho}$ such that $a_{2}$ is invertible on the support of $\phi$, and choose $B$ such that

$$
b\left(x, \xi^{\prime}\right)=\phi\left(x, \xi^{\prime}\right) a_{2}^{-1} \tau^{2}
$$

The equality (3.10) then shows that $\left\langle\mu, \mathbb{1}_{\mathcal{G}} \phi\right\rangle=0$, which completes the proof using the positivity of $\mu$.

\subsection{The propagation theorem}

\subsubsection{The generalized bicharacteristic flow}

The characteristic curves of the Hamiltonian flow of $p$

$$
H_{p}=\partial_{\xi} p \partial_{x}-\partial_{x} p \partial_{\xi}
$$

define a local flow on $T^{*} X$. The symbol $p$ is homogeneous of degree 2 in $\xi$, so that the flow of $H_{p}$ does not yield a flow on the quotient space $S_{b}^{*} X$. To get such a flow, we shall replace $p$ by $p / \tau$ which is homogeneous of degree 1. Note that on the support of $\mu$ (where $\tau$ does not vanish), $p$ is null, so that $\frac{1}{\tau} H_{p}$ and $H_{p / \tau}$ are equal. Furthermore, the integral curves of $\frac{1}{\tau} H_{p}$ and $H_{p}$ are the same.

Let $\Sigma$ be a small conic open subset of $Z=j($ Char $P)$. Set $q_{0}:=q_{\left\lceil x_{n}=0\right.}, q_{1}:=\partial_{x_{n}} q_{\left\lceil x_{n}=0\right.}$ and

$$
\begin{gathered}
\Sigma^{0}:=\Sigma \cap\left\{x_{n}>0\right\} \\
\Sigma^{1}:=\mathcal{H}=\Sigma \cap\left\{x_{n}=0, q_{0}<0\right\} \\
\Sigma^{2}:=\Sigma \cap\left\{x_{n}=0, q_{0}=0, q_{1} \neq 0\right\} \\
\Sigma^{k+3}:=\Sigma \cap\left\{x_{n}=0, q_{0}=q_{1}=\ldots=H_{q_{0}}^{k} q_{1}=0, H_{q_{0}}^{k+1} q_{1} \neq 0\right\} .
\end{gathered}
$$

Assume that in $\Sigma$, there is no contact of infinite order between the bicharacteristic curves of $P$ and the boundary, which means that for a certain finite integer $J$

$$
\exists J \in \mathbb{N}, \quad \Sigma=\bigcup_{j \leq J} \Sigma^{J}
$$

Decompose $\Sigma^{2}$ in the disjoint union

$$
\Sigma^{2}=\mathcal{G}^{2,+} \cup \mathcal{G}^{2,-}, \quad \mathcal{G}^{2,+}:=\Sigma^{2} \cap\left\{q_{1}<0\right\}, \mathcal{G}^{2,-}:=\Sigma^{2} \cap\left\{q_{1}>0\right\}
$$

The set $\mathcal{G}^{2,+}$ is the set of strictly diffractive points and $\mathcal{G}^{2,-}$ the set of strictly gliding points.

Definition 3.9. Let $\gamma$ be a map from a real interval $I$ to $\Sigma$ and

$$
\Gamma(s)=j^{-1}(\gamma(s)) \in S \text { Char } P
$$

which is defined as long as $\gamma(s) \notin \mathcal{H}$. Such a map $\gamma(s)=(x(s), \xi(s))$ is called a ray, or a general bicharacteristic curve when $\gamma$ is continuous from $I$ to $\Sigma$ and for all $s_{0}$ in $I$,

- if $x_{n}\left(s_{0}\right)>0, \Gamma$ is differentiable in $s_{0}$ and

$$
\Gamma^{\prime}\left(s_{0}\right)=\frac{1}{\tau} H_{p} \Gamma\left(s_{0}\right)
$$


- if $\gamma\left(s_{0}\right) \in \mathcal{H} \cup \mathcal{G}^{2,+}$,

$$
\exists \varepsilon>0, \quad \forall s \in] s_{0}-\varepsilon, s_{0}[\cup] s_{0}, s_{0}+\varepsilon\left[, \quad x_{n}(s)>0\right.
$$

- if $\gamma\left(s_{0}\right) \in \mathcal{G} \backslash \mathcal{G}^{2,+}, \Gamma$ is well defined and differentiable near $s_{0}$ and

$$
\Gamma^{\prime}\left(s_{0}\right)=\frac{1}{\tau} H_{q_{0}} \Gamma\left(s_{0}\right)
$$

(Thus, if $\gamma$ stays in this region, its spatial projection is a geodesic of the boundary.)

Under the assumption (3.11), R. Melrose and J. Sjöstrand have shown that for any $\rho \in \Sigma$, there exists a unique maximal ray $\gamma$ taking values in $\Sigma$ such that $\gamma(0)=\rho$ (cf. [17], [11], Chap. 24.3). In the sequel, we shall denote by $\phi(s, \rho)$ the resulting flow (satisfying $\phi(0, \rho)=\rho)$. The function $p / \tau$ being homogeneous of degree 1 in $\xi, \phi$ also defines a flow on $\Sigma / \mathbb{R}_{+}^{*}$.

\subsubsection{The uniform Lopatinsky conditions}

Notations. Let $S_{\partial}^{m}$ be the set of symbols $a\left(x^{\prime}, \xi^{\prime}\right)$ of pseudo-differential operators on $\partial X$, with compact support in $x^{\prime}$, with principal symbol homogeneous of degree $m$ in $\xi^{\prime}$, and $\mathcal{A}_{\partial}^{m}$ the set of corresponding compactly supported pseudo-differential operators ( $c f$. Sect. 3.1 .3 for precise definitions). The Sobolev spaces on $\partial X$, defined as those on $X$, shall be denoted by $\boldsymbol{H}_{\partial}^{s}, \boldsymbol{H}_{\mathrm{loc}, \partial}^{s}, \boldsymbol{H}_{\tilde{\rho}, \partial}^{s}$.

An approximate pseudo-differential equation on the traces of $u^{k}$ is said to satisfy Lopatinsky conditions when it is independent of the equation $P u^{k}=0$. More precisely:

Definition 3.10. Under the assumptions (3.1) and (3.2), the sequence $\left(u^{k}\right)$ is said to satisfy uniform Lopatinsky boundary conditions at $\tilde{\rho} \in S^{*} \partial X$ when

- if $\tilde{\rho} \in \mathcal{G}, \exists B_{-1} \in \mathcal{A}_{\partial}^{-1}$ such that

$$
\left\{\begin{array}{c}
u_{\uparrow x_{n}=0}^{k}=B_{-1}\left(D_{x_{n}} u_{\uparrow x_{n}=0}^{k}\right)+h^{k} \\
h_{k \rightarrow+\infty}^{k} \underset{\text { in } \boldsymbol{H}_{\tilde{\rho}, \partial}^{1}}{\longrightarrow}
\end{array}\right.
$$

- if $\tilde{\rho} \in \mathcal{H}, \exists B_{0} \in \mathcal{A}_{\partial}^{0}$ such that

$$
\left\{\begin{array}{c}
D_{x_{n}} u_{\uparrow x_{n}=0}^{k}-\Lambda u_{\uparrow x_{n}=0}^{k}=B_{0}\left(D_{x_{n}} u_{\uparrow x_{n}=0}^{k}+\Lambda u_{\uparrow x_{n}=0}^{k}\right)+h^{k} \\
\sigma\left(B_{0}\right)(\tilde{\rho}) \text { invertible, } \quad h^{k} \underset{k \rightarrow+\infty}{\longrightarrow} 0 \text { in } \boldsymbol{L}_{\tilde{\rho}, \partial}^{2} \\
\Lambda \in \mathcal{A}_{\partial}^{1}, \quad \sigma(\Lambda)=\sqrt{q_{0}\left(x^{\prime}, \xi^{\prime}\right)}=\sqrt{\nu^{2} \tau^{2}-\left\|\eta^{\prime}\right\|^{2}} \text { near } \tilde{\rho}
\end{array}\right.
$$

( $\left\|\eta^{\prime}\right\|^{2}={ }^{t} \eta^{\prime} g^{\prime-1} \eta^{\prime}$ is the natural Euclidean norm in the local coordinate system).

Examples. - The Dirichlet boundary condition, $u_{\mid x_{n}=0}^{k}=0$, or more generally a pseudo-differential boundary condition of the form

$$
\begin{aligned}
& u_{\uparrow x_{n}=0}^{k}=B_{-1} D_{x_{n}} u_{\uparrow x_{n}=0}^{k}+h^{k}, \quad B_{-1} \in \mathcal{A}^{-1} \\
& h^{k} \underset{k \rightarrow+\infty}{\longrightarrow} 0 \text { in } \boldsymbol{H}_{\tilde{\rho}, \partial}^{1} \text {, }
\end{aligned}
$$

where the eigenvalues of $\sigma\left(B_{-1}\right)$ are all pure imaginary numbers near $\tilde{\rho}$, is a uniform Lopatinsky boundary condition, whether $\tilde{\rho}$ is glancing or hyperbolic. 
- The Neumann condition

$$
D_{x_{n}} u_{\uparrow x_{n}=0}^{k} \underset{k \rightarrow+\infty}{\longrightarrow} 0 \text { in } \boldsymbol{L}_{\tilde{\rho}, \partial}^{2}
$$

is a uniform Lopatinsky condition near any $\tilde{\rho} \notin \mathcal{G}$, but not at $\tilde{\rho} \in \mathcal{G}$.

In the glancing case, a boundary condition of the form (3.12) implies that $\left(u^{k}\right)$ is regular near the boundary close to $\tilde{\rho}$ (see Prop. 3.18), so that we can define the microlocal defect measure $\mu$ of $u^{k}$, and the bicharacteristic flow is defined $\mu$-almost everywhere in a neighbourhood of $\tilde{\rho}$ (since $\mu(\mathcal{E})=0$ ).

The set $\mathcal{G}^{2,+}$ is transverse to the bicharacteristic flow. The following result (proved in [3], Sect.3.4) is needed in the next paragraph to treat the propagation of $\mu$ by the flow.

Lemma 3.11. Suppose that on $\Sigma, u^{k}$ satisfies uniform Lopatinsky boundary conditions. Then

$$
\mu\left(\mathcal{G}^{2,+} \cap \Sigma\right)=0
$$

\subsubsection{The propagation theorem}

When a uniform Lopatinsky condition holds, $\mu$ propagates along the integral curves of the bicharacteristic flow. In the hyperbolic region, there is a jump (which depends upon the boundary condition). We shall only state a propagation theorem for the support of $\mu$, without giving a complete description of the propagation of $\mu$.

Theorem 3.12. Let $\tilde{\rho} \in \mathcal{H} \cap \mathcal{G}$ such that $\left(u^{k}\right)$ satisfies Lopatinsky boundary conditions. Consider a small conic open neighbourhood $\Sigma$ of $\tilde{\rho}$ in $S \hat{Z}$ such that on $\Sigma$, (3.12) (or (3.12')) holds. Then the support of $\mu$ is, in $\Sigma$, invariant by the bicharacteristic flow.

(cf. [3], Chap. 3.3, Thm. 1)

In other terms, if $\rho \in \Sigma$ is on the support of $\mu$, so is the entire bicharacteristic passing through $\rho$ in $\Sigma$.

Remark 3.13. Inside $M$, Theorem 3.12 is an easy consequence of the transport equation on $\mu$ :

$$
\langle\mu,\{p / \tau, a\}\rangle, \quad a \in C_{0}^{\infty}\left(Z \cap\left\{x_{n}>0\right\}\right),
$$

which may be immediately derived, using symbolic calculus, from the property

$$
\lim _{k \rightarrow+\infty}\left(A_{1} P u^{k}-P A_{1} u^{k}, u^{k}\right)=0, \quad A_{1} \in \mathcal{A}_{i}^{1}
$$

obtained by integration by parts with the equation (3.5). Near a boundary point, property (3.15), with $A_{1} \in \mathcal{A}_{b}^{1}$, still holds with an additional boundary term. Consequently, (3.14) holds only for a certain class of function $a \in C_{0}^{\infty}(S Z)$, satisfying a particular boundary condition on $\left\{x_{n}=0\right\}$ (condition chosen so that the boundary term vanishes when $k$ goes to $\infty$ ). The proof of the propagation theorem, which is fairly technical, uses (3.14), and near strictly diffractive points, Lemma 3.11. The boundary condition on $a$ gives the exact value of the jump in the hyperbolic region. See par. 3 of [3] for details.

\subsection{Estimates on traces}

We now state precise properties of the traces of $u^{k}$ in the hyperbolic, elliptic and glancing regions, which are one of the main tools of the proofs of the following sections. Those results are fairly classical, and we only shall give a proof (in the appendix) for the glancing case. See Appendix of [3] for proofs in the hyperbolic and elliptic cases. In this paragraph, we shall always assume $\left(u^{k}\right)$ satisfies (3.1) and (3.2). 


\subsubsection{Hyperbolic region}

Near an hyperbolic point, one gains without any boundary condition, half a derivative in comparison with the standard traces theorem.

Proposition 3.14. Let $\tilde{\rho} \in \mathcal{H}$. Then

$$
u_{\uparrow x_{n}=0}^{k} \underset{k \rightarrow \infty}{=} O(1) \text { in } \boldsymbol{H}_{\tilde{\rho}, \partial}^{1}, \quad \partial_{x_{n}} u_{\uparrow x_{n}=0}^{k} \underset{k \rightarrow \infty}{=} O(1) \text { in } \boldsymbol{L}_{\tilde{\rho}, \partial}^{2} .
$$

In view of the propagation theorem in the interior of $M$, the support of $\mu$ is, near $\tilde{\rho}$, the union of incoming rays (integral curves of $H_{p / \tau}$ along which $\xi_{n}<0$ ) and outgoing rays (integral curves of $H_{p / \tau}$ along which $\left.\xi_{n}>0\right)$. When the sequence satisfies uniform Lopatinsky conditions, Theorem 3.12 is equivalent to the fact that if an incoming (respectively outgoing) ray is in the support of $\mu$, so is the outgoing (respectively incoming) ray passing through the same hyperbolic point. In the opposite case where the support of $\mu$ contains, locally, only incoming (or only outgoing) rays, one gets a boundary condition which is orthogonal to the uniform Lopatinsky conditions:

Proposition 3.15. Assume that near $\tilde{\rho} \in \mathcal{H}$, on the support of $\mu, \xi_{n}>0$. Then

$$
D_{x_{n}} u_{\uparrow x_{n}=0}^{k}+\Lambda u_{\uparrow x_{n}=0}^{k}=o(1) \text { in } \boldsymbol{L}_{\tilde{\rho}, \partial}^{2}, \quad \Lambda \in \mathcal{A}_{\partial}^{1}, \quad \sigma_{1}(\Lambda)=\sqrt{\nu^{2} \tau^{2}-\left\|\eta^{\prime}\right\|^{2}}
$$

On the other hand, if near $\tilde{\rho} \in \mathcal{H}$, on the support of $\mu, \xi_{n}<0$, then

$$
D_{x_{n}} u_{\uparrow x_{n}=0}^{k}-\Lambda u_{\uparrow x_{n}=0}^{k}=o(1) \text { in } \boldsymbol{L}_{\tilde{\rho}, \partial}^{2} .
$$

In particular, if $\mu$ is null near $\tilde{\rho}$,

$$
D_{x_{n}} u_{\uparrow x_{n}=0}^{k} \underset{k \rightarrow+\infty}{\longrightarrow} 0 \text { in } \boldsymbol{L}_{\tilde{\rho}, \partial}^{2}, \quad u_{\uparrow x_{n}=0}^{k} \underset{k \rightarrow+\infty}{\longrightarrow} 0 \text { in } \boldsymbol{H}_{\tilde{\rho}, \partial}^{1} .
$$

\subsubsection{In the elliptic region}

In $\mathcal{E}$, the equation (3.5) implies a pseudo-differential traces equation on $u^{k}$ :

Proposition 3.16. Let $\tilde{\rho} \in \mathcal{E}$ and $\left(u^{k}\right)$ satisfy the assumptions of Theorem 3.4. Let $M>0$. Then

$$
\begin{gathered}
D_{x_{n}} u_{\uparrow x_{n}=0}^{k}+\Xi u_{\uparrow x_{n}=0}^{k} \underset{k \rightarrow+\infty}{\longrightarrow} 0 \text { in } H_{\tilde{\rho}}^{M} \\
\Xi \in \mathcal{A}^{1}, \quad \sigma_{1}(\Xi)=i \sqrt{q_{0}}=i \sqrt{\left\|\eta^{\prime}\right\|^{2}-\nu^{2} \tau^{2}} \text { near } \tilde{\rho} .
\end{gathered}
$$

In particular, if a boundary condition independent of (3.16) holds on $u^{k}$ near $\tilde{\rho}$ (such a condition is called as in the glancing and hyperbolic cases a uniform Lopatinsky condition), the traces of $u^{k}$ converge to 0 in $H_{\tilde{\rho}}^{M}$. Proposition 3.16 still holds in a much more general case, for example if $P$ is replaced by a non-scalar operator $\mathbf{P}$. The principal symbol of $\Xi$ depends again upon the principal symbol of $\mathbf{P}$. In the next proposition, we only state a consequence of this fact when $\left(u^{k}\right)$ satisfies Dirichlet boundary conditions (which are of uniform Lopatinsky type).

Proposition 3.17. Let $\mathbf{P}:=D_{x_{n}}^{2}+\mathbf{Q}_{1} D_{x_{n}}+\mathbf{Q}_{2}$, where each $\mathbf{Q}_{\mathbf{j}}$ is a matrix pseudo-differential operator of degree $j$, with principal symbol $\mathbf{q}_{j}$. Let $\mathbf{p}(x, \xi):=\xi_{n}^{2}+\mathbf{q}_{2}+\xi_{n} \mathbf{q}_{1}$ be the principal symbol of $\mathbf{P}$, and $\tilde{\rho}=\left(\tilde{x}^{\prime}, \tilde{\xi}^{\prime}\right)$ be a point of $S^{*} \partial X$ such that the matrix $\mathbf{p}\left(\tilde{x}^{\prime}, 0, \tilde{\xi}^{\prime}, \xi_{n}\right)$ is invertible for any real number $\xi_{n}$. Consider a sequence $\left(u^{k}\right)$, weakly converging to 0 in $\boldsymbol{H}_{\mathrm{loc}}^{1}(\bar{X})$ and satisfying

$$
\mathbf{P} u^{k}=0, \quad u_{\left\lceil x_{n}=0\right.}^{k}=0
$$


Then for all $M$,

$$
\partial_{x_{n}} u_{\left\lceil x_{n}=0\right.}^{k} \underset{k \rightarrow+\infty}{\longrightarrow} 0 \text { in } \boldsymbol{H}_{\tilde{\rho}, \partial}^{M} \text {. }
$$

We shall later apply the preceding proposition in the elliptic zone $\left(\mathcal{E}_{T} \cap \mathcal{E}_{L}\right.$ with notations of Sect. 3.5.2) of the Lamé operator $\partial_{t}^{2}-\Delta_{e}$.

\subsubsection{In the glancing region}

The strong results of the two preceding paragraphs do not hold in the neighbourhood of a glancing point. In this case, one needs boundary conditions to get better estimates than the standard traces theorem with loss of one half-derivative. In the case of Lopatinsky boundary conditions, the results are similar to those of the hyperbolic region.

Proposition 3.18. a) Let $\tilde{\rho} \in \mathcal{G}$. Assume that $\left(u^{k}\right)$ satisfies Lopatinsky uniform boundary conditions near $\tilde{\rho}$. Then

$$
u_{\uparrow x_{n}=0}^{k}=O(1) \text { in } \boldsymbol{H}_{\tilde{\rho}, \partial}^{1}, \quad \partial_{x_{n}} u_{\uparrow x_{n}=0}^{k}=O(1) \text { in } \boldsymbol{L}_{\tilde{\rho}, \partial}^{2} \cdot
$$

Furthermore, if $\mu$ vanishes near $\tilde{\rho}$, then

$$
u_{\uparrow x_{n}=0}^{k}=o(1) \text { in } \boldsymbol{H}_{\tilde{\rho}, \partial}^{1}, \quad \partial_{x_{n}} u_{\uparrow x_{n}=0}^{k}=o(1) \text { in } \boldsymbol{L}_{\tilde{\rho}, \partial}^{2} .
$$

b) Assume

$$
u_{\uparrow x_{n}=0}^{k}=o(1) \text { in } \boldsymbol{H}_{\tilde{\rho}, \partial}^{1}, \quad \partial_{x_{n}} u_{\uparrow x_{n}=0}^{k}=o(1) \text { in } \boldsymbol{L}_{\tilde{\rho}, \partial}^{2},
$$

and that every $\tilde{\rho} \in \mathcal{G}$ is not diffractive, in the sense that at least one off the two half-bicharacteristic passing through $\tilde{\rho}$ stays in $\partial \Omega$ near $\tilde{\rho}$. Then $\mu=0$ near $\tilde{\rho}$.

Remark 3.19. As seen in Propositions 3.14 and 3.15, point a) holds in the hyperbolic case, where no boundary condition is required.

The proof of Proposition 3.18 is given in the appendix.

\subsection{The Lamé system}

This subsection is devoted to the Lamé system with Dirichlet boundary conditions on an open bounded subset $\Omega$ of $\mathbb{R}^{3}$ :

$$
\left\{\begin{array}{c}
\partial_{t}^{2} u-\Delta_{e} u=0,(t, y) \in \mathbb{R} \times \Omega \\
u_{\uparrow \partial \Omega}=0 \\
u_{\uparrow t=0}=u_{0} \in \boldsymbol{H}_{0}^{1}, \partial_{t} u_{\uparrow t=0}=u_{1} \in \boldsymbol{L}^{2} .
\end{array}\right.
$$

In Section 3.5.1, (3.20) is decomposed into two wave equations. In Section 3.5.2, we shall introduce the defect measures associated to these equations. Next paragraphs are devoted to a few elementary properties of these measures.

We will often work in local coordinates. As before (but with $n=3$ ) we will only use geodesic normal coordinates near the boundary, choosing the coordinate $x_{3}$ to be the distance to the boundary $\left\{x_{3}=0\right\}$. 


\subsubsection{Transversal and longitudinal waves}

The natural energy

$$
E(t):=\frac{1}{2} \int_{\Omega}\left(\left|\partial_{t} u\right|^{2}+\varsigma|\nabla u|^{2}+(\lambda+\varsigma)|\operatorname{div} u|^{2}\right) \mathrm{d} y
$$

is time-invariant. Let $E_{0}$ be its constant value. The next classical proposition is proved, for example, in [3]. Recall that $M:=\mathbb{R} \times \Omega$.

Proposition 3.20 (Decomposition of the Lamé system). There exists a constant $C>0$ such that for every solution $u$ of (3.20), there exists

$$
u_{T} \in \boldsymbol{H}_{\mathrm{loc}}^{1}(\bar{M}), u_{L} \in \boldsymbol{H}_{\mathrm{loc}}^{1}(\bar{M}),
$$

such that

1. $u=u_{T}+u_{L}, \operatorname{div} u_{T}=0, \operatorname{curl} u_{L}=0$.

2. $\left(\partial_{t}^{2}-c_{T}^{2} \Delta\right) u_{T}=0$, where $c_{T}^{2}:=\varsigma$.

3. $\left(\partial_{t}^{2}-c_{L}^{2} \Delta\right) u_{L}=0$, where $c_{L}^{2}:=\lambda+2 \varsigma$.

4. For every bounded interval I of $\mathbb{R}$, of length $|I|$,

$$
\left\|u_{L}\right\|_{\boldsymbol{H}^{1}(I \times \Omega)}^{2}+\left\|u_{T}\right\|_{\boldsymbol{H}^{1}(I \times \Omega)}^{2} \leq C|I| E_{0} .
$$

5. If $u_{0}$ and $u_{1}$ are linear combinations of a finite number of eigenfunctions of $\Delta_{e}$, then

$$
u_{T} \in C^{\infty}(\bar{M}), \quad u_{L} \in C^{\infty}(\bar{M}) .
$$

Definition 3.21. The function $u_{T}$ is called the transversal wave, and $u_{L}$ the longitudinal wave.

Remark 3.22. In the sequel we shall often reduce the longitudinal wave to a scalar function, writing $u_{L}=\nabla \varphi$, with

$$
\varphi \in \boldsymbol{H}_{\mathrm{loc}}^{2}(\bar{M}), \quad\|\varphi\|_{\boldsymbol{H}^{2}(I \times \Omega)} \leq C|I| E_{0}, \quad\left(\partial_{t}^{2}-c_{L}^{2} \Delta\right) \varphi=0 .
$$

\subsubsection{Measures}

Let $\left(u^{k}\right)$ be a sequence of solutions of the Lamé system with

$$
\left(u_{0}^{k}, u_{1}^{k}\right) \underset{k \rightarrow+\infty}{\longrightarrow} 0 \text { in } \boldsymbol{H}_{0}^{1}(\Omega) \times \boldsymbol{L}^{2}(\Omega) .
$$

In view of Proposition 3.20, both sequences $\left(u_{T}^{k}\right)$ and $\left(u_{L}^{k}\right)$ weakly converge to 0 in $\boldsymbol{H}_{\mathrm{loc}}^{1}(\mathbb{R} \times \bar{\Omega})$. Likewise, the sequence $\left(\partial_{t} \varphi^{k}\right)$ weakly converges to 0 in $H_{\text {loc }}^{1}\left(\mathbb{R} \times \bar{\Omega}\right.$ ) (we consider a derivative of $\varphi$ in order to work in a $H^{1}$ space since we have defined the defect measures in such spaces).

Lemma 3.23. The sequences $\left(u_{T}^{k}\right),\left(u_{L}^{k}\right),\left(\partial_{t} \varphi^{k}\right)$ are regular on the boundary.

(cf. [3], Lem. 4.2)

Notations. Let

- $\mu_{T}, \mu_{L}$ and $\mu$ be the defect measures respectively associated (up to a subsequence) to $\left(u_{T}^{k}\right),\left(u_{L}^{k}\right)$, and $\left(\partial_{t} \varphi^{k}\right)$ by Theorem 3.4; 
- $\mathcal{H}_{T}, \mathcal{H}_{L}, \mathcal{G}_{T}, \mathcal{G}_{L}, \mathcal{E}_{T}, \mathcal{E}_{L}$ the hyperbolic, glancing and elliptic region of the transversal and longitudinal waves.

All the calculation shall be carried out in one of the $J+1$ local coordinate systems chosen in the beginning of this section. We shall make a distinction between the spaces of scalar operators $\mathcal{A}^{m}, \mathcal{A}_{\partial}^{m}$ (defined in Sect. 3.1.3, Sect. 3.1.4, Sect. 3.1.8, with $N=1$ ), and the spaces of matrix operators $\mathcal{A}^{m}, \mathcal{A}_{\partial}^{m}$ (with $N=3$ ).

The notation $x=(t, y)$ always refers to local coordinates. When a distinction is necessary, we shall write coordinates on $\Omega$ before the change of variables $z=\left(z_{1}, z_{2}, z_{3}\right)$. This global system of coordinates has been chosen so that the magnetic field $\boldsymbol{B}$ has coordinates $(B, 0,0)$.

Remark 3.24. The condition $c_{L}^{2} \neq c_{T}^{2}$ means that the intersection of $\mathcal{G}_{T}$ and $\mathcal{G}_{L}$ is empty.

\subsubsection{Link between $\mu$ and $\mu_{L}$}

In a fixed coordinate patch, let $\chi$ be the local diffeomorphism from global spatial coordinates $\left(z_{1}, z_{2}, z_{3}\right)$ to local coordinates:

$$
z=\chi(y), \quad{ }^{t} \chi^{\prime}(y) \zeta=\eta
$$

\section{Proposition 3.25.}

$$
\forall a \in C_{0}^{0}\left(S_{b}^{*}(\mathbb{R} \times \bar{\Omega}), M^{3}(\mathbb{C})\right),\left\langle\mu, \frac{{ }^{t} \zeta a \zeta}{\tau^{2}}\right\rangle=\left\langle\mu_{L}, a\right\rangle
$$

In particular, the measures $\mu$ and $\mu_{L}$ have the same supports.

Proof. Let $A_{j} \in \mathcal{A}^{j}, j=1,2, \quad A:=A_{-1} D_{x_{3}}+A_{0}$.

Set $I_{k}:=\left(A \partial_{t} u_{L}^{k}, \partial_{t} u_{L}^{k}\right)$.

On one hand,

$$
I_{k}=-\left(\partial_{t} A \partial_{t} u_{L}^{k}, u_{L}^{k}\right) \underset{k \rightarrow+\infty}{\longrightarrow}\left\langle\mu_{L}, a\right\rangle .
$$

On the other hand,

$$
I_{k}=-\left(\operatorname{div} A \nabla \partial_{t} \varphi^{k}, \partial_{t} \varphi^{k}\right)+o(1) \underset{k \rightarrow+\infty}{\longrightarrow}\left\langle\mu, \frac{{ }^{t} \zeta a \zeta}{\tau^{2}}\right\rangle
$$

The boundary terms of this preceding integration by parts converge to 0 according to Lemma 3.23. This implies Proposition 3.25 when $a$ is of the form $\xi_{3} a^{-1}+a_{0}$, and then by a density argument for any $a$.

\subsubsection{Polarization of $\mu_{T}$ and $\mu_{L}$}

Let $\pi$ be the orthogonal projection in $\mathbb{C}^{3}$ on the line spanned by $\zeta \in \mathbb{R}^{3}$, and $\pi_{\perp}$ the orthogonal projection on the plane normal to $\zeta$.

$$
\pi(V):=|\zeta|^{-2}\left({ }^{t} \zeta . V\right) \zeta, \quad \pi_{\perp}:=\operatorname{Id}_{\mathbb{C}^{3}}-\pi .
$$

The projectors $\pi$ and $\pi_{\perp}$ are defined by the formulas (3.21) on $S^{*} \bar{X}$.

Proposition 3.26. The measure $\mu_{L}$ is polarized along the direction of propagation, and $\mu_{T}$ orthogonally to this direction:

$$
\mu_{L}=\pi \mu_{L} \pi, \quad \mu_{T}=\pi_{\perp} \mu_{T} \pi_{\perp} .
$$


Proof. The statement on $\mu_{L}$ is an immediate consequence of Proposition 3.25. To show the statement on $\mu_{T}$, take $A_{0} \in \mathcal{A}_{0}$. The nullity of $\operatorname{div} u_{T}^{k}$ implies

$$
0=\left(A_{0} \nabla \operatorname{div} u_{T}^{k}, u_{T}^{k}\right) \underset{k \rightarrow+\infty}{\longrightarrow}\left\langle\mu_{T}, \frac{a_{0} \zeta^{t} \zeta}{\tau^{2}}\right\rangle
$$

Thus $<\mu_{T}, a \pi>=0$ i.e. $\left\langle\mu_{T}, a \pi_{\perp}>=<\mu_{T}, a>\right.$. The symmetry of $\mu_{T}$ completes the proof.

Remark 3.27. To get more intrinsic formulations of the preceding results, i.e. statements where the two coordinate system do not mix, one should have considered $u_{L}^{k}$ and $u_{T}^{k}$ as section of the tangent space $T \Omega$, and defined measures with values endomorphism of $T \Omega$ (instead of endomorphism of $\mathbb{C}^{3}$ ).

\subsubsection{A decoupling lemma}

The next result, converting an approximate differential equation on $u^{k}$ into two equations on $u_{L}^{k}$ and $u_{T}^{k}$, is of crucial importance in the sequel. As before, (.,.) stands for the $\boldsymbol{L}^{2}$ scalar product on $\mathbb{R} \times \Omega$.

Lemma 3.28 (decoupling lemma). Let $A$ be a pseudo-differential operator of order 2 of the following form

$$
A=\sum_{j=-M}^{2} A_{j} \partial_{x_{3}}^{2-j}+A_{i}, \quad A_{i} \in \mathcal{A}_{i}^{2}, \quad A_{j} \in \mathcal{A}^{j}
$$

Then

$$
\lim _{k \rightarrow+\infty}\left(A u_{T}^{k}, u_{L}^{k}\right)=\lim _{k \rightarrow+\infty}\left(A u_{L}^{k}, u_{T}^{k}\right)=0
$$

Let $A$ be a $(1,3)$ matrix of pseudo-differential operators, with coefficients of the form (3.22), but with scalar operators. Then

$$
\lim _{k \rightarrow+\infty}\left(A u_{T}^{k}, \partial_{t} \varphi^{k}\right)=0
$$

Proof. We shall only prove the convergence to 0 of $\left(A u_{T}^{k}, u_{L}^{k}\right)$. The proof of rest of the lemma is very much the same. We may obviously assume that the operators $A_{j}$ have compact support in one of the local coordinate system introduced in Section 3.1.8. In view of the equations

$$
-\mathrm{g}^{-1 / 2} \partial_{x_{3}} \mathrm{~g}^{1 / 2} \partial_{x_{3}} u_{T}^{k}+Q_{T} u_{T}^{k}=0, \quad-\mathrm{g}^{-1 / 2} \partial_{x_{3}} \mathrm{~g}^{1 / 2} \partial_{x_{3}} u_{L}^{k}+Q_{L} u_{L}^{k}=0
$$

where $Q_{T}$ and $Q_{L}$ are tangential, it is sufficient to prove the lemma in the cases $j=1,2$ and in the interior case.

First case: $A \in \mathcal{A}^{i}$.

Since $\nu_{L} \neq \nu_{T}$ it is easy to construct two operators $\Psi_{T}, \Psi_{L} \in \mathcal{A}_{i}^{0}$ such that

$$
\left(\Psi_{T}+\Psi_{L}\right)_{\uparrow U}=\mathrm{Id}, \quad \operatorname{supp} \sigma_{0}\left(\Psi_{T}\right) \cap \mathcal{Z}_{L}=\operatorname{supp} \sigma_{0}\left(\Psi_{L}\right) \cap \mathcal{Z}_{T}=\emptyset,
$$

where $U$ is an open subset of $\Omega$ such that there exists a function $\varphi \in C_{0}^{\infty}(U)$ satisfying $\varphi A \varphi=A$. Writing

$$
A=A \varphi=A \Psi_{L} \varphi+A \Psi_{T} \varphi,
$$


we may assume that the principal symbol of $A$ does not intersect $\mathcal{Z}_{T}$ (or does not intersect $\mathcal{Z}_{L}$ ). For such operators, (3.23) holds has a direct consequence of Proposition 3.2. For example, in the first case we have

$$
A u_{T}^{k}=O(1) \text { in } \boldsymbol{L}^{2}
$$

Second case: $A \in \mathcal{A}_{\partial}^{2}$.

We know that the support of $\mu_{T} \mathbb{1}_{\left\{x_{3}=0\right\}}$ is included in $\mathcal{G}_{T}$ and the support of $\mu_{L} \mathbb{1}_{\left\{x_{3}=0\right\}}$ in $\mathcal{G}_{L}$.

As a consequence, we may write $A=A\left(\Theta_{T}+\Theta_{L}\right)$ where $\Theta_{T}$ and $\Theta_{L}$ are tangential operators of degree 0 such that

$$
\operatorname{supp} \sigma_{0}\left(\Theta_{T}\right) \cap \mathcal{G}_{L}=\operatorname{supp} \sigma\left(\Theta_{L}\right) \cap \mathcal{G}_{T}=\emptyset
$$

We may thus assume that the support of the principal symbol $a_{2}$ of $A$ is disjoint from one of the two glancing sets, say $\mathcal{G}_{T}$. We have

$$
\left(A u_{T}^{k}, u_{L}^{k}\right)=\left(\chi\left(x_{3} / \varepsilon\right) A u_{T}^{k}, u_{L}^{k}\right)+\left(\left(1-\chi\left(x_{3} / \varepsilon\right)\right) A u_{T}^{k}, u_{L}^{k}\right) .
$$

where $\chi$ is a compactly supported function in $\mathbb{R}$ equal to 1 near the origin. We first fix $\varepsilon$ and let $k$ tend to $\infty$. The second term of the sum tends to 0 in view of the preceding case. As for the first term, we have, $u_{L}^{k}$ being bounded in $\boldsymbol{H}^{1}$,

$$
\begin{aligned}
\left|\left(\chi\left(x_{3} / \varepsilon\right) A u_{T}^{k}, u_{L}^{k}\right)\right| & \leq C\left\|\chi\left(x_{3} / \varepsilon\right) A u_{T}^{k}\right\|_{L^{2}\left(0, l, \boldsymbol{H}^{-1}\left(X^{\prime}\right)\right)}\left\|u_{L}^{k}\right\|_{L^{2}\left(0, l, \boldsymbol{H}^{1}\left(X^{\prime}\right)\right)} \\
& \leq C\left\|\Lambda_{-1}^{\prime} \chi\left(x_{3} / \varepsilon\right) A u_{T}^{k}\right\|_{\boldsymbol{L}^{2}}+o(1), k \rightarrow+\infty,
\end{aligned}
$$

where $\Lambda_{-1}^{\prime} \in \mathcal{A}_{\partial}^{-1}$, with principal symbol equal to $\left\|\xi^{\prime}\right\|^{-1}$ near the support of $A$. We have

$$
\begin{gathered}
\left\|\Lambda_{-1}^{\prime} \chi\left(x_{3} / \varepsilon\right) A u_{T}^{k}\right\|_{L^{2}}^{2} \underset{k \rightarrow+\infty}{\longrightarrow}\left\langle\mu_{T}, \frac{\left(\chi\left(x_{3} / \varepsilon\right)\right)^{2}\left|a_{2}\right|^{2}}{\tau^{2}\left\|\xi^{\prime}\right\|^{2}}\right\rangle \\
\limsup _{k \rightarrow+\infty}\left|\left(A u_{T}^{k}, u_{L}^{k}\right)\right| \leq\left\langle\mu_{T}, \frac{\left(\chi\left(x_{3} / \varepsilon\right)\right)^{2}\left|a_{2}\right|^{2}}{\tau^{2}\left\|\xi^{\prime}\right\|^{2}}\right\rangle .
\end{gathered}
$$

When $\varepsilon$ goes to 0 , the right side of this inequality converges (by the dominated convergence theorem) to

$$
\left\langle\mu_{T}, \mathbb{1}_{\left\{x_{3}=0\right\}}\left|a_{2}\right|^{2} \tau^{-2}\left\|\xi^{\prime}\right\|^{-2}\right\rangle
$$

which is null, because $\mathcal{G}_{T}$ and the support of $a_{2}$ are disjoint.

Third case: $A=A_{1} D_{x_{3}}, A_{1}$ tangential.

As in the preceding case, we may assume that $\sigma_{1}\left(A_{1}\right)$ is disjoint from one of the two glancing sets, say $\mathcal{G}_{T}$. Then

$$
\left(A u_{T}^{k}, u_{L}^{k}\right)=\left(\chi\left(x_{3} / \varepsilon\right) A u_{T}^{k}, u_{L}^{k}\right)+\left(\left(1-\chi\left(x_{3} / \varepsilon\right)\right) A u_{T}^{k}, u_{L}^{k}\right)
$$

The second term converges to zero when $k \rightarrow \infty$ for the same reasons as in the case $A \in \mathcal{A}_{i}^{2}$. The first term may be written

$$
\begin{aligned}
\left(\chi\left(x_{3} / \varepsilon\right) A_{1} D_{x_{3}} u_{T}^{k}, u_{L}^{k}\right) & =\left(D_{x_{3}}\left(\chi\left(x_{3} / \varepsilon\right) A_{1} u_{T}^{k}\right), u_{L}^{k}\right)+\left(R_{\varepsilon} u_{T}^{k}, u_{L}^{k}\right), R_{\varepsilon} \in \mathcal{A}^{1} \\
& =\left(\left(\chi\left(x_{3} / \varepsilon\right) A_{1} u_{T}^{k}\right), D_{x_{3}} u_{L}^{k}\right)+\left(R_{\varepsilon} u_{T}^{k}, u_{L}^{k}\right)+\{\text { boundary terms }\}
\end{aligned}
$$


The boundary terms tend to zero when $k$ tends to infinity because $\left(u_{T}^{k}\right)$ and $\left(u_{L}^{k}\right)$ are regular on the boundary. The proof may be completed as in the preceding case, letting $k$ go to infinity then $\varepsilon$ go to zero.

\section{Sufficient CONDITION}

If $\gamma$ is a $\boldsymbol{B}$-resistant ray defined on a real interval $] a, b[$, we shall call life-length the positive quantity $\mid t(b)-$ $t(a) \mid$. In this section we use the tools introduced in the preceding section to prove the following:

Proposition 4.1. Let $T>0$. Assume that every $\boldsymbol{B}$-resistant ray in $\Omega$ is of life-length strictly less than $T$. Then there exists $C>0$ such that for every solution of the Lamé system (3.20)

$$
\left\|u_{0}\right\|_{\boldsymbol{H}_{0}^{1}}^{2}+\left\|u_{1}\right\|_{\boldsymbol{L}^{2}}^{2} \leq C\left(\left\|\operatorname{curl}\left(\partial_{t} u \wedge \boldsymbol{B}\right)\right\|_{\boldsymbol{H}^{-1}((0, T) \times \Omega)}^{2}+\left\|u_{0}\right\|_{\boldsymbol{L}^{2}}^{2}+\left\|u_{1}\right\|_{\boldsymbol{H}^{-1}}^{2}\right) .
$$

Inequality (4.1) is the sufficient condition (2.8) for uniform decay stated in point a) of Proposition 2.5. Proposition 4.1 thus completes the proof of the sufficient condition of Theorem 1.2, namely that the nonexistence of arbitrarily large $\boldsymbol{B}$-resistant rays on $\Omega$ implies the uniform decay of the energy for solutions of the system of magnetoelasticity.

To show (4.1), we shall argue by contradiction, considering the defect measures $\mu_{T, L}$ of Section 3.5.2 associated to a sequence $\left(u^{k}\right)$ which contradicts (4.1) (cf. Sect. 4.1). The bound on $\operatorname{curl}\left(\partial_{t} u^{k} \wedge \boldsymbol{B}\right)$ given by the negation of (4.1) implies a strong condition on the supports of these measures (see Sect. 4.2). In Section 4.3, we make use of this condition, together with propagation arguments near the boundary of $\Omega$. Section 4.4 completes the proof, using the assumption of non-existence of $\boldsymbol{B}$-resistant rays of life-length larger than $\mathrm{T}$.

\subsection{Introduction of measures}

Assume that (4.1) does not hold. Then there exists a sequence $\left(u^{k}\right)$ of solutions of the Lamé system such that

$$
1=\left\|u_{0}^{k}\right\|_{\boldsymbol{H}_{0}^{1}}^{2}+\left\|u_{1}^{k}\right\|_{\boldsymbol{L}^{2}}^{2}>k\left(\left\|\operatorname{curl}\left(\partial_{t} u^{k} \wedge \boldsymbol{B}\right)\right\|_{\boldsymbol{H}^{-1}((0, T) \times \Omega)}^{2}+\left\|u_{0}^{k}\right\|_{\boldsymbol{L}^{2}}^{2}+\left\|u_{1}^{k}\right\|_{\boldsymbol{H}^{-1}}^{2}\right) .
$$

Up to the extraction of a subsequence, one may assume that $\left(u_{0}^{k}, u_{1}^{k}\right)$ weakly converges in $\boldsymbol{H}_{0}^{1} \times \boldsymbol{L}^{2}$. Inequality (4.2) implies that its weak limit is 0. We may thus introduce the defect measures $\mu_{T}, \mu_{L}$ and $\mu$ of Section 3.5.2, associated to the sequences $\left(u_{T}^{k}\right),\left(u_{L}^{k}\right)$ and $\left(\partial_{t} \varphi^{k}\right)$. To contradict (4.2), we need to show that these measures are null. Note that (4.2) implies

$$
\operatorname{curl}\left(\partial_{t} u^{k} \wedge \boldsymbol{B}\right) \underset{k \rightarrow+\infty}{\longrightarrow} 0 \text { in } H^{-1}((0, T) \times \Omega) .
$$

Remark 4.2. By a density argument, it suffices to show (4.1) with $\left(u_{0}, u_{1}\right)$ generated by a finite number of eigenfunctions of $\mathcal{L}$. We may thus assume, that $u_{L}^{k}$ and $u_{T}^{k}$ are $C^{\infty}$.

Remark 4.3. We will indeed show a more precise statement than Proposition 4.1 namely that if $\left(u^{k}\right)$ is a sequence of solutions of the Lamé system converging weakly to 0 in the energy space and satisfying (4.3) then the set $\left(\operatorname{supp} \mu_{T} \cup \operatorname{supp} \mu_{L}\right) \cap\{t \in(0, T)\}$ a union of $\boldsymbol{B}$-resistant rays of length $T$.

\subsection{Condition on the supports}

We may see $\boldsymbol{B}$ as a vector field on $\Omega$, i.e. a section of $T \Omega$. To avoid confusions, the magnetic field considered as a vector field shall be referred as $\vec{B}$. In a local coordinate system, if

$$
\chi: U \subset \bar{\Omega} \longrightarrow \overline{\mathbb{R}}_{+}^{3}
$$


is the change of coordinates, and $\chi^{\prime}$ its differential, $\vec{B}$ is equal to $\chi^{\prime} \boldsymbol{B}$. Notation $\boldsymbol{B}$ shall always refer to the vector of $\mathbb{R}^{3}$ of coordinates $(B, 0,0)$. As before, $\left(z_{1}, z_{2}, z_{3}\right)$ refers to the global spatial coordinates on $\Omega$, before the change of variable.

Lemma 4.4. Assume (4.3). Then, on the interval $(0, T)$,

$$
\begin{array}{r}
\mu_{T} \mathbb{1}_{(0, T)}=\mu_{T} \mathbb{1}_{(0, T)} \mathbb{1}_{\vec{B}^{\perp}}, \quad \vec{B}^{\perp}:=\left\{(t, y, \tau, \eta) ;{ }^{t} \vec{B} \eta=0\right\} \\
\mu_{L} \mathbb{1}_{(0, T)}=\mu_{L} \mathbb{1}_{(0, T)} \mathbb{1}_{\vec{B} / /}, \quad \vec{B} / /:=\{(t, y, \tau, \eta) ; \eta \in \operatorname{vect}(g \vec{B})\} .
\end{array}
$$

Proof. Set

$$
R u:=\operatorname{curl}\left(\partial_{t} u \wedge \vec{B}\right)=B \partial_{t}\left(\begin{array}{c}
-\partial_{z_{2}} u_{2}-\partial_{z_{3}} u_{3} \\
\partial_{z_{1}} u_{2} \\
\partial_{z_{1}} u_{3}
\end{array}\right)
$$

Transversal measure. The measure $\mu_{T}$ does not charge neither $\mathcal{H}_{T}$ nor $\mathcal{E}_{T}$. Thus, it suffices to check

$$
\operatorname{supp} \mu_{T} \mathbb{1}_{(0, T)} \mathbb{1}_{\left\{x_{3}>0\right\} \cup \mathcal{G}_{T}} \subset \vec{B}^{\perp} .
$$

Near the boundary, by Proposition A.1 of the appendix,

$$
R u^{k} \underset{k \rightarrow+\infty}{\longrightarrow} 0 \text { in } L^{2}\left(\left[0, l\left[, \boldsymbol{H}_{\mathrm{loc}}^{-1}\left(X^{\prime}\right)\right) .\right.\right.
$$

Thus, according to the decoupling lemma,

$$
\forall A_{0} \in \mathcal{A}^{0}, \operatorname{supp} A_{0} \subset\{t \in(0, T)\} \Longrightarrow\left(A_{0} R u_{T}^{k}, u_{T}^{k}\right) \underset{k \rightarrow+\infty}{\longrightarrow} 0 .
$$

Because $\operatorname{div} u_{T}^{k}=0$, the definition (4.6) of $R u_{T}$ implies $R u_{T}^{k}=\partial_{t} \partial_{z_{1}} u_{T}^{k}$. By Section 3.2.2,

$$
\mu_{T}\left(\{t \in(0, T)\} \cap\left\{\sigma_{2}\left(\partial_{t} \partial_{z_{1}}\right) \neq 0\right\}\right)=0 .
$$

This clearly shows the announced result near an interior point. When $\tilde{\rho} \in \mathcal{G}_{T}$ one may write $\partial_{z_{1}}=f_{0} \partial_{x_{3}}+F_{1}$, where $f_{0}$ is a function and $F_{1}$ a first order tangential differential operator, and the following basic fact completes the proof:

$$
\sigma\left(F_{1}\right)(\tilde{\rho})=0 \Longleftrightarrow \tilde{\eta}^{\prime} \perp \vec{B}
$$

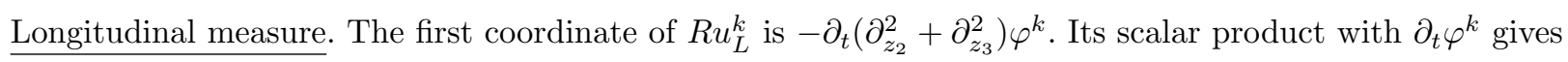

$$
\forall A_{0} \in \mathcal{A}^{0}, \operatorname{supp}\left(A_{0}\right) \subset\{t \in(0, T)\} \Longrightarrow \lim _{k \rightarrow+\infty}\left(A_{0}\left(\partial_{z_{2}}^{2}+\partial_{z_{3}}^{2}\right) \partial_{t} \varphi^{k}, \partial_{t} \varphi^{k}\right)=0
$$

This implies (again by Sect. 3.2.2), that $\mu$ (thus $\mu_{L}$ ) vanishes, in $(0, T)$, on the set of all $\rho$ such that

$$
\rho \in\left\{x_{3}>0\right\} \cup \mathcal{G}_{T}, \quad \sigma_{2}\left(\partial_{z_{2}}^{2}+\partial_{z_{3}}^{2}\right)(\rho) \neq 0 .
$$

Hence (4.5). 


\subsection{Support of the measure near the boundary}

For any symbol $q_{0}$ with support in $\left\{x_{3}>0\right\}$, we have

$$
\left\langle\mu_{T}, H_{p_{T} / \tau} q_{0}\right\rangle=0 \quad\left\langle\mu_{L}, H_{p_{L} / \tau} q_{0}\right\rangle=0,
$$

which shows, in the interior of $\Omega$, the invariance of each measure by the associated Hamiltonian flow. Unfortunately, the condition

$$
u_{T \mid \partial \Omega}^{k}+u_{L\lceil\partial \Omega}^{k}=0
$$

is not always sufficient to extend such a property in the neighbourhood of $\partial \Omega$. Indeed, $\mu_{L}$ and $\mu_{T}$ are not deterministic for general sequences of solutions of the Lamé system, in the sense that the value of the two measures for time $t>t_{0}$ is not uniquely determined by their value for time $t \leq t_{0}$. In our case, this convenient property holds thanks to the strong conditions on the support of $\mu_{T}$ and $\mu_{L}$. As announced before, we shall only describe the propagation of the supports of the measure.

Lemma 4.5. Let $\mu_{T}$ and $\mu_{L}$ be the defect measures associated to a sequence of solutions of the Lamé system satisfying (4.3). Let

$$
\tilde{\rho}=\left(\tilde{x}^{\prime}, \tilde{\xi}^{\prime}\right)=\left(\tilde{t}, \tilde{y}^{\prime}, \tilde{\tau}, \tilde{\eta}^{\prime}\right) \in S^{*} \partial X
$$

and $\mathbf{n}$ the unitary exterior normal vector to $\partial \Omega$ at $\tilde{y}^{\prime}$. Them $\mu_{T}$ and $\mu_{L}$ both vanish near $\tilde{\rho}$ except possibly in the following cases (cf. Fig. 2):

1. $\mu_{L}$ is null. The support of $\mu_{T}$ propagates along the transversal flow and

- $\left(\mathcal{H}_{T[1]}\right)$ case: $\tilde{\rho} \in \mathcal{H}_{T}, \tilde{\eta}^{\prime}=0$ and $\vec{B}$ is orthogonal to $\mathbf{n}$;

- $\overline{\left(\mathcal{H}_{T[2]}\right) \text { case }}: \tilde{\rho} \in \mathcal{H}_{T}, \tilde{\eta}^{\prime} \neq 0$ and $\vec{B}$ is normal to the reflection plane;

- $\left(\mathcal{G}_{\left.T^{[1]}\right) \text { case: }} \tilde{\rho}\right.$ is diffractive for the transversal wave (i.e. $\tilde{\rho} \in \mathcal{G}_{T}$ and the bicharacteristic ray passing through $\tilde{\rho}$ intersects the boundary only at $\tilde{\rho})$, and $\vec{B}$ is orthogonal to $\tilde{\eta}^{\prime}$;

- $\left(\mathcal{G}_{T^{[2]}}\right)$ case: $\tilde{\rho} \in \mathcal{G}_{T}$ is not diffractive for the transversal wave, and $\vec{B}$ is normal to the reflection plane.

2. $\mu_{T}$ is null, the support of $\mu_{L}$ propagates along the longitudinal flow and

- $\left(\mathcal{H}_{L}\right)$ case: $\tilde{\rho} \in \mathcal{H}_{L}, \tilde{\eta}^{\prime}=0$ and $\vec{B}$ is parallel to $\mathbf{n}$;

- $\overline{\left(\mathcal{G}_{L}\right) \text { case: }} \tilde{\rho}$ is a diffractive point for the longitudinal wave and $\vec{B}$ is parallel to $\tilde{\eta}^{\prime}$.

3. Both measures $\mu_{T}$ and $\mu_{L}$ are non null, $\tilde{\rho} \in \mathcal{H}_{T} \cap \mathcal{H}_{L}$ and

- $(T \rightarrow L)$ case: $\vec{B}$ is orthogonal to the transversal ray coming in, and parallel to the longitudinal ray going out of $\tilde{\rho}$. The support of $\mu_{T}$ is a union of incoming transversal rays. The support of $\mu_{L}$ is the union of all outgoing longitudinal rays going out of points of $\mathcal{H}_{T} \cap \mathcal{H}_{L}$ where the transversal rays of the support of $\mu_{T}$ come in;

- $(L \rightarrow T)$ case: $\vec{B}$ is orthogonal to the transversal ray going out of and parallel to the longitudinal ray coming in $\tilde{\rho}$. The support of $\mu_{L}$ is a union of incoming longitudinal rays. The support of $\mu_{T}$ is the union of all outgoing transversal rays going out of points of $\mathcal{H}_{T} \cap \mathcal{H}_{L}$ where the longitudinal rays of the support of $\mu_{L}$ come in.

All the assertions of Lemma 4.5 should be understood in a neighbourhood of $\tilde{\rho}$. The reflection plane at a boundary point $\rho=\left(t, y^{\prime}, \tau, \eta^{\prime}\right)$, defined as long as $\eta^{\prime} \neq 0$, is the plane passing through $y^{\prime}$ and generated by $\mathbf{n}$ and $\eta^{\prime}$, thus containing the bicharacteristic ray passing through $\rho$. The statement " $\vec{B}$ is parallel to $\eta^{\prime \prime}$ must be understood as "the vector $g \vec{B}$ of the cotangent bundle of $\Omega$ is parallel to $\eta^{\prime \prime}$. 


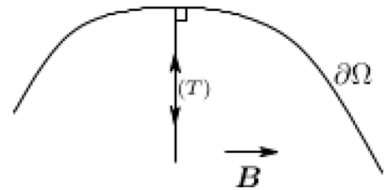

$\left(\mathcal{H}_{T[1]}\right)$

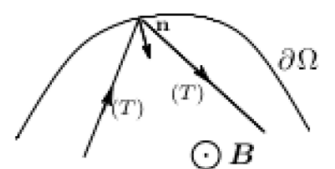

$\left(\mathcal{H}_{T[2]}\right)$

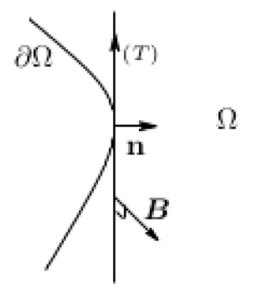

$\left(\mathcal{G}_{T[1]}\right)$

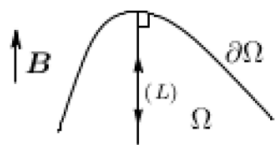

$\left(\mathcal{H}_{L}\right)$

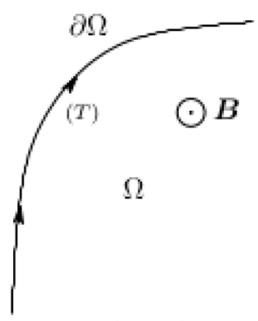

$\left(\mathcal{G}_{T[2]}\right)$

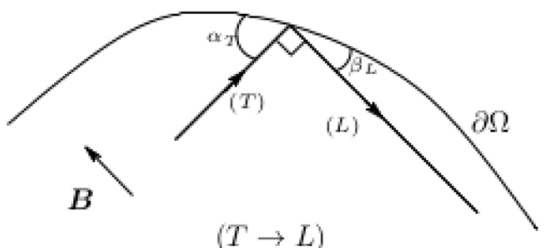

$(T \rightarrow L)$

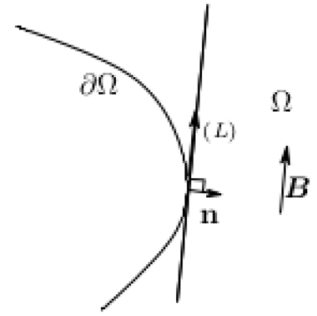

$\left(\mathcal{G}_{L}\right)$

Figure 2. Cases arising in Lemma 4.5.

Notation. Let $\rho$ be an hyperbolic point for the transversal (respectively longitudinal) wave. We shall denote by $\xi_{T}^{-}, \xi_{T}^{+}$(respectively $\xi_{L}^{-}, \xi_{L}^{+}$) the incoming and outgoing vectors through $\rho$ :

$$
\xi_{T}^{+}:=\left(\begin{array}{c}
\xi^{\prime} \\
\xi_{T 3}=\sqrt{\nu_{T}^{2} \tau^{2}-\left\|\eta^{\prime}\right\|^{2}}
\end{array}\right) \quad \xi_{T}^{-}=\left(\begin{array}{c}
\xi^{\prime} \\
-\xi_{T 3}
\end{array}\right)
$$

(respectively with " $L$ " instead of " $T$ ").

We shall write $\eta_{T}^{ \pm}, \eta_{L}^{ \pm}$the spatial components of this vectors. For example,

$$
\eta_{T}^{+}=\left(\frac{\eta^{\prime}}{\sqrt{\nu_{T}^{2} \tau^{2}-\left\|\eta^{\prime}\right\|^{2}}}\right)
$$

Let us postpone the proof of Lemma 4.5 to show, as stated in the introduction of this article, that in the $(T \rightarrow L)$ and $(L \rightarrow T)$ cases, the angles of refraction and incidence have a fixed value, determined by the quotient $c_{T} / c_{L}$. Consider for example the $(T \rightarrow L)$ case. Let $\alpha_{T}$ be the angle of incidence of the transversal wave, $\beta_{L}$ the angle of refraction of the longitudinal wave, $a_{T}$ and $a_{L}$ the following numbers:

$$
a_{T}:=\tan \alpha_{T}=\frac{\tilde{\xi}_{T 3}}{\left\|\tilde{\eta}^{\prime}\right\|}, \quad a_{L}:=\tan \beta_{L}=\frac{\tilde{\xi}_{L 3}}{\left\|\tilde{\eta}^{\prime}\right\|} .
$$


The incident and refracted waves are orthogonal, so that

$$
\left\|\tilde{\eta}^{\prime}\right\|^{2}-\tilde{\xi}_{T 3} \tilde{\xi}_{L 3}=0 \text {, i.e. } a_{T} a_{L}=1 \text {. }
$$

Furthermore, the definitions of $\tilde{\eta}_{L 3}^{-}$and $\tilde{\eta}_{T 3}^{+}$yield

$$
c_{T}^{2}\left\|\tilde{\eta}_{T}^{+}\right\|^{2}-\tau^{2}=0, \quad c_{L}^{2}\left\|\tilde{\eta}_{L}^{-}\right\|^{2}-\tau^{2}=0,
$$

which gives the equation

$$
c_{T}^{2}\left(1+a_{T}^{2}\right)=c_{L}^{2}\left(1+a_{L}^{2}\right) .
$$

Equations (4.9) and (4.10) imply the formula announced in the introduction:

$$
\alpha_{T}=\arctan \frac{c_{L}}{c_{T}}, \beta_{L}=\arctan \frac{c_{T}}{c_{L}} .
$$

By a similar calculation, one gets, in the $(T \rightarrow L)$ case, $\alpha_{L}=\arctan \frac{c_{T}}{c_{L}}, \beta_{T}=\arctan \frac{c_{L}}{c_{T}}$.

Proof of Lemma 4.5. We start with case (3): $\mu_{T} \neq 0, \mu_{L} \neq 0$ near $\tilde{\rho}$. In this case, $\tilde{\rho} \notin \mathcal{E}_{T} \cup \mathcal{E}_{L}$. It is also easy to show that $\tilde{\rho} \notin \mathcal{G}_{T} \cup \mathcal{G}_{L}$. Indeed, if $\tilde{\rho} \in \mathcal{G}_{T}$ then it also belongs to $\mathcal{H}_{L}$ (it cannot be a point of $\mathcal{E}_{L}$, and $\mathcal{G}_{L}$ and $\mathcal{G}_{T}$ are disjoint). But $\mu_{T}$ being non-null near $\tilde{\rho}, \tilde{\eta}$ is orthogonal to $\vec{B}$ (by Lem. 4.4) so neither $\tilde{\eta}_{L}^{+}$nor $\tilde{\eta}_{L}^{-}$are parallel to $\vec{B}$, which implies (again by Lem. 4.4) that $\mu_{L}=0$ near $\tilde{\rho}$, contradicting our assumptions. Likewise, if $\tilde{\rho} \in \mathcal{G}_{L}, \tilde{\eta}^{\prime}$ must be parallel to $\vec{B}$ and $\mu_{T}$ null near $\tilde{\rho}$. Thus $\tilde{\rho} \in \mathcal{H}_{T} \cap \mathcal{H}_{L}$. The support of measures $\mu_{T}$ and $\mu_{L}$ is, near $\tilde{\rho}$, a union of incoming and outgoing maximal rays.

Let us first assume that the support of $\mu_{L}$ contains the ray going out of $\tilde{\rho}$. Then

$$
\tilde{\eta}_{L}^{+} / / \vec{B}
$$

so that $\tilde{\eta}_{T}^{+}$is not orthogonal to $\vec{B}$. As a consequence, the support of $\mu_{T}$ is only made of incoming rays, and the fact that $\mu_{T} \neq 0$ implies

$$
\tilde{\eta}_{T}^{-} \perp \vec{B}
$$

Thus $\tilde{\eta}_{L}^{-}$is not parallel to $\vec{B}$. This is the $(T \rightarrow L)$ case, and it remains to show the statement of the lemma about the transfer from transversal incoming waves to longitudinal outgoing waves, which may be formulated as follow: for any $\breve{\rho} \in \mathcal{H}_{T} \cap \mathcal{H}_{L}$ near $\tilde{\rho}$ the following equivalence holds:

$$
\breve{\rho} \in \operatorname{supp} \mu_{T} \Longleftrightarrow \breve{\rho} \in \operatorname{supp} \mu_{L} .
$$

Let us assume for example $\breve{\rho} \notin \operatorname{supp} \mu_{T}$. Then $\mu_{T}$ is null near rays coming in and going out of $\breve{\rho}$ and by the hyperbolic theory (see Prop. 3.15),

$$
u_{L\left\lceil x_{3}=0\right.}^{k}=-u_{T\left\lceil x_{3}=0\right.}^{k} \underset{k \rightarrow+\infty}{\longrightarrow} 0 \text { in } \boldsymbol{L}_{\breve{\rho}, \partial}^{2} .
$$

By the propagation theorem, the support of $\mu_{L}$ propagates near $\breve{\rho}$. But this support is a union of outgoing rays. Consequently, it is empty near $\breve{\rho}$ and $\mu_{L}$ is null near $\breve{\rho}$. The implication $\breve{\rho} \notin \operatorname{supp} \mu_{L} \Longrightarrow \breve{\rho} \notin \operatorname{supp} \mu_{T}$ may be shown in the same manner. 
If the support of $\mu_{L}$ contains no outgoing ray near $\tilde{\rho}$, it must contain incoming rays. This corresponds to the $(L \rightarrow T)$ case, which may be treated as the $(T \rightarrow L)$ case.

The study of the other cases is based on the transfer of the boundary conditions stated in Lemma 4.6 below: roughly, a boundary condition on the longitudinal wave implies one on the transversal wave and vice versa.

Notation. Let $v_{T, L}^{k}$ be the functions $u_{T, L}^{k}$ considered as vector fields on $\Omega$. In local coordinates, if $\chi$ denotes the change of coordinates, we have

$$
v_{T}^{k}=\chi^{\prime}(y) u_{T}^{k}=\left(\begin{array}{c}
v_{T 1}^{k} \\
v_{T 2}^{k} \\
v_{T 3}^{k}
\end{array}\right) \quad v_{L}^{k}=\chi^{\prime}(y) u_{L}^{k}=\left(\begin{array}{c}
v_{L 1}^{k} \\
v_{L 2}^{k} \\
v_{L 3}^{k}
\end{array}\right) .
$$

Lemma 4.6. Let $\tilde{\rho} \in S^{*} \partial X$, and $\left(u^{k}\right)$ be any sequence of solutions of the Lamé system weakly converging to 0 in $\boldsymbol{H}_{\mathrm{loc}}^{1}(\mathbb{R} \times \bar{\Omega})$.

- Assume the following approximate equation for some $A_{1} \in \mathcal{A}^{1}$ :

$$
\partial_{x_{3}} v_{T 3\left\lceil x_{3}=0\right.}^{k}=A_{1} v_{T 3\left\lceil x_{3}=0\right.}^{k}+o(1) \text { in } L_{\tilde{\rho}, \partial}^{2} .
$$

Then

$$
\Delta_{y^{\prime}} \varphi_{\left\lceil x_{3}=0\right.}^{k}=-A_{1} \partial_{x_{3}} \varphi_{\left\lceil x_{3}=0\right.}^{k}+o(1) \text { in } L_{\tilde{\rho}, \partial}^{2} .
$$

- Conversely, if, for some $A_{-1} \in \mathcal{A}^{-1}$ the following equation holds:

$$
\varphi_{\left\lceil x_{3}=0\right.}^{k}=A_{-1} \partial_{x_{3}} \varphi_{\left\lceil x_{3}=0\right.}^{k}+o(1) \text { in } H_{\tilde{\rho}, \partial}^{2} .
$$

Then

$$
\partial_{x_{3}} v_{T 3\left\lceil x_{3}=0\right.}^{k}=-\Delta_{y^{\prime}} A_{-1} v_{T 3\left\lceil x_{3}=0\right.}^{k}+o(1) \text { in } L_{\tilde{\rho}, \partial}^{2} .
$$

- Moreover if, in addition to (4.13), $\tilde{\eta}^{\prime} \neq 0$ and $\sigma\left(A_{-1}\right)(\tilde{\rho}) \neq 0$, then

$$
\begin{aligned}
& v_{T\left\lceil x_{3}=0\right.}^{k}=\mathbf{Z}_{-1} \partial_{x_{3}} v_{T\left\lceil x_{3}=0\right.}^{k}+o(1) \text { in } \boldsymbol{H}_{\tilde{\rho}, \partial}^{1} \\
& \mathbf{Z}_{-1} \in \mathcal{A}^{-1}, \sigma_{-1}\left(\mathbf{Z}_{-1}\right)=\left\|\eta^{\prime}\right\|^{-2}\left(\begin{array}{ccc}
0 & 0 & i g^{-1}\left[\begin{array}{c}
\eta_{1} \\
\eta_{2}
\end{array}\right] \\
0 & 0 & 0 \\
0 & 0 & \sigma\left(A_{-1}\right)^{-1}
\end{array}\right) \text {. }
\end{aligned}
$$

Proof. First note that the Dirichlet condition on $u^{k}$ implies

$$
\partial_{x_{3}} v_{T 3\left\lceil x_{3}=0\right.}^{k}=\Delta_{y^{\prime}} \varphi_{\left\lceil x_{3}=0\right.}^{k}+O(1) \text { in } H_{\mathrm{loc}}^{1 / 2}(\partial X),
$$

Indeed, the equation $\operatorname{div} u_{T}^{k}=0$ implies, recalling that $\operatorname{div} v_{T}^{k}=\frac{1}{\sqrt{\mathrm{g}}} \sum_{j=1}^{3} \partial_{y_{j}}\left(\sqrt{\mathrm{g}} v_{T j}^{k}\right)$.

$$
\partial_{x_{3}} v_{T 3}^{k}+\partial_{y_{1}} v_{T 1}^{k}+\partial_{y_{2}} v_{T 2}^{k}=O(1) \text { in } H_{\mathrm{loc}}^{1}(X) .
$$

Using $v_{3\left\lceil x_{3}=0\right.}^{k}=0$, we obtain

$$
\partial_{x_{3}} v_{T 3\left\lceil x_{3}=0\right.}^{k}=\partial_{y_{1}} v_{L 1\left\lceil x_{3}=0\right.}^{k}+\partial_{y_{2}} v_{L 2\left\lceil x_{3}=0\right.}^{k}+O(1) \text { in } H_{\mathrm{loc}}^{1 / 2}(\partial X),
$$


yielding (4.14) by the definition of $\varphi$.

Assume (4.12). By (4.14) and the nullity of $v_{3\left\lceil x_{3}=0\right.}^{k}$,

$$
\Delta_{y^{\prime}} \varphi_{\left\lceil x_{3}=0\right.}^{k}=-A_{1} v_{L 3\left\lceil x_{3}=0\right.}^{k}+o(1)=-A_{1} \partial_{x_{3}} \varphi_{\left\lceil x_{3}=0\right.}^{k}+o(1) \text { in } \boldsymbol{L}_{\tilde{\rho}, \partial}^{2} .
$$

Now assume (4.13). Then $\Delta_{y^{\prime}} \varphi_{\left\lceil x_{3}=0\right.}^{k}=\Delta_{y^{\prime}} A_{-1} \partial_{x_{3}} \varphi_{\left\lceil x_{3}=0\right.}^{k}+o(1)$ in $L_{\tilde{\rho}, \partial}^{2}$, which implies (4.13') using (4.14) on the left-hand side of the equation, and the Dirichlet condition on $v_{3}^{k}$ on its right-hand side.

If, in addition to the assumption (4.13), $\tilde{\eta}^{\prime}$ and $\sigma\left(A_{-1}\right)\left(\tilde{\rho}^{\prime}\right)$ are nonzero, both operators $A_{-1}$ and $\Delta_{y^{\prime}}$ are elliptic at $\tilde{\rho}$, and equations (4.13') and (4.14) may be rewritten

$$
\begin{array}{ll}
v_{T 3\left\lceil x_{3}=0\right.}^{k}=Y_{-1} \partial_{x_{3}} v_{T 3\left\lceil x_{3}=0\right.}^{k}+o(1) \text { in } H_{\tilde{\rho}, \partial}^{1} & \sigma\left(Y_{-1}\right)=\left\|\eta^{\prime}\right\|^{-2} \sigma\left(A_{-1}\right)^{-1}, \\
\varphi_{\left\lceil x_{3}=0\right.}^{k}=E_{-2} \partial_{x_{3}} v_{T 3\left\lceil x_{3}=0\right.}^{k}+O(1) \text { in } H_{\tilde{\rho}, \partial}^{5 / 2}, & \sigma\left(E_{-2}\right)=-\left\|\eta^{\prime}\right\|^{-2} \text { near } \tilde{\rho}
\end{array}
$$

Hence

$$
\begin{gathered}
\left(\begin{array}{c}
v_{T 1\left\lceil x_{3}=0\right.}^{k} \\
v_{T 2\left\lceil x_{3}=0\right.}^{k}
\end{array}\right)=g^{\prime-1}\left(\begin{array}{c}
\partial_{y_{1}} \varphi_{\left\lceil x_{3}=0\right.}^{k} \\
\partial_{y_{2}} \varphi_{\left\lceil x_{3}=0\right.}^{k}
\end{array}\right)=Z_{-1} \partial_{x_{3}} v_{T 3\left\lceil x_{3}=0\right.}^{k}+O(1) \text { in } H_{\tilde{\rho}, x_{3}=0}^{1 / 2} \\
\sigma\left(Z_{-1}\right)=i\left\|\eta^{\prime}\right\|^{-2} g^{\prime-1}\left(\begin{array}{c}
\eta_{1} \\
\eta_{2}
\end{array}\right) \text { near } \tilde{\rho} .
\end{gathered}
$$

Assertion (4.13") is an easy consequence of (4.15) and (4.16).

We may now study cases (1) and (2) of Lemma 4.5.

Case (1): assume $\mu_{L}=0, \mu_{T} \neq 0$ near $\tilde{\rho}$. There are three possibilities:

- If $\tilde{\rho} \in \mathcal{H}_{L}$, the nullity of $\mu_{L}$ implies, by standard hyperbolic theory (Prop. 3.15),

$$
u_{T\left\lceil x_{3}=0\right.}^{k}=-u_{L\left\lceil x_{3}=0\right.}^{k} \underset{k \rightarrow+\infty}{\longrightarrow} 0 \text { in } \boldsymbol{H}_{\tilde{\rho}, \partial}^{1},
$$

so that near $\tilde{\rho}$, the support of $\mu_{T}$ propagates. It is easy to see that condition (4.4) on the support of $\mu_{T}$ implies, if $\mu_{T}$ does not vanish, that this is one of the four cases described in Lemma 4.5, (1) .

- Assume $\tilde{\rho} \in \mathcal{E}_{L}$. The standard elliptic theory (Prop. 3.16) implies

$$
\partial_{x_{3}} \varphi_{\left\lceil x_{3}=0\right.}^{k}+i \Xi \varphi_{\left\lceil x_{3}=0\right.}^{k} \underset{k \rightarrow+\infty}{\longrightarrow} 0 \text { in } H_{\tilde{\rho},\left\lceil x_{3}=0\right.}^{1},
$$

where $\sigma_{1}(\Xi)=i \sqrt{\left\|\eta^{\prime}\right\|^{2}-\nu_{L}^{2} \tau^{2}}$. With Lemma 4.6, this yields the following equation on the traces of $u_{T}^{k}$ :

$$
u_{T\left\lceil x_{3}=0\right.}^{k}=i \widetilde{\mathbf{Z}}_{-1} D_{x_{3}} u_{T\left\lceil x_{3}=0\right.}^{k}+o(1) \text { in } \boldsymbol{H}_{\tilde{\rho}, \partial}^{1}, \quad \widetilde{\mathbf{Z}}_{-1}=\chi^{\prime-1} \mathbf{Z}_{-1} \chi^{\prime}
$$

where the principal symbol of the operator $\mathbf{Z}_{-1} \in \mathcal{A}^{-1}$ is given by (4.13"), with $\sigma_{-1}\left(A_{-1}\right)^{-1}=-i \sigma_{1}(\Xi)$. Notice that the eigenvalues of $\sigma_{-1}\left(i \mathbf{Z}_{-1}\right)$, thus those of $\sigma_{-1}\left(i \widetilde{\mathbf{Z}}_{-1}\right)$ are pure imaginary numbers. As a consequence, the boundary condition (4.17) is a uniform Lopatinsky boundary condition near $\tilde{\rho}$ (see the example following Def. 3.10), which shows again the propagation of $\mu_{T}$. As in the case where $\tilde{\rho} \in \mathcal{H}_{L}$, it is easy to see that this is one of the four cases of Lemma 4.5.

- The case $\tilde{\rho} \in \mathcal{G}_{L}$ is the most difficult. If $\mu_{T}$ is non-null near $\tilde{\rho}$, then $\tilde{\rho}$ must be in $\mathcal{H}_{T}$. We use a contradiction argument to prove the propagation of the support of $\mu_{T}$. Let $\breve{\rho} \in \mathcal{H}_{T}$ such that the ray coming in $\breve{\rho}$ is in the 
support of $\mu_{T}$, but not the ray going out of $\breve{\rho}$. According to the standard hyperbolic theory (Prop. 3.15),

$$
D_{x_{3}} u_{T\left\lceil x_{3}=0\right.}^{k}-\Lambda_{T} u_{T\left\lceil x_{3}=0\right.}^{k} \underset{k \rightarrow+\infty}{\longrightarrow} 0 \text { in } \boldsymbol{L}_{\tilde{\rho}, \partial}^{2}, \quad \sigma_{1}\left(\Lambda_{T}\right)=\sqrt{\nu_{T} \tau^{2}-\left\|\eta^{\prime}\right\|^{2}}
$$

This implies, by Lemma 4.6, a boundary equation on $\partial_{t} \varphi^{k}$, of the following form:

$$
\partial_{t} \varphi_{\left\lceil x_{3}=0\right.}^{k}=Y_{-1} D_{x_{3}} \partial_{t} \varphi_{\uparrow x_{3}=0}^{k}+o(1) \text { in } H_{\breve{\rho}, \partial}^{1},
$$

which is a uniform Lopatinsky condition near $\breve{\rho}$ because $\tilde{\rho} \in \mathcal{G}_{L}$. In view of Proposition 3.18 on traces in the glancing region, such an equation implies, with the nullity of $\mu$ near $\breve{\rho}$ the following conditions:

$$
\partial_{t} \varphi_{\uparrow_{3}=0}^{k} \rightarrow 0 \text { in } H_{\breve{\rho}, \partial}^{1}, \quad \partial_{n} \partial_{t} \varphi_{\uparrow_{3}=0}^{k} \rightarrow 0 \text { in } L_{\breve{\rho}, \partial}^{2} .
$$

The operator $\partial_{t}$ being elliptic at $\breve{\rho}$, this shows that $u_{L}^{k}$ tends to 0 in $\boldsymbol{H}_{\breve{\rho}, \partial}^{1}$, and thus

$$
u_{T\left\lceil x_{3}=0\right.}^{k} \underset{k \rightarrow+\infty}{\longrightarrow} 0 \text { in } \boldsymbol{H}_{\breve{\rho}, \partial}^{1} .
$$

Hence the propagation of the support of $\mu_{T}$ near $\breve{\rho}$, which contradicts the assumption on rays coming in and going out of $\breve{\rho}$.

Similar arguments show that if the ray going out of $\breve{\rho}$ is in the support of $\mu_{T}$, so is the ray coming in $\breve{\rho}$. This proves that the support of $\mu_{T}$ propagates near $\tilde{\rho}$. Notice that this is necessarily the $\left(\mathcal{H}_{\left.T^{[2]}\right]}\right)$ case.

Case 2: we assume now that $\mu_{T}=0$ and $\mu_{L} \neq 0$. We argue in a similar way, considering three possibilities:

- If $\tilde{\rho} \in \mathcal{H}_{T}$, the standard hyperbolic theory gives an approximate Dirichlet boundary equation on $u_{T}^{k}$, which implies

$$
u_{L\left\lceil x_{3}=0\right.}^{k} \underset{k \rightarrow+\infty}{\longrightarrow} 0 \text { in } \boldsymbol{H}_{\tilde{\rho}, \partial}^{1} .
$$

As a consequence, the support of $\mu_{L}$ propagates. On this support, $\eta$ is parallel to $\vec{B}$, which shows, as stated in Lemma 4.5 , that $\tilde{\eta^{\prime}}=0$ and $\tilde{\rho} \in \mathcal{H}_{L}$, or $\tilde{\eta^{\prime}} / / \vec{B}$ and $\tilde{\rho} \in \mathcal{G}_{L}$.

- If $\tilde{\rho} \in \mathcal{E}_{T}$, we write (as in the similar situation when $\tilde{\rho} \in \mathcal{E}_{L}$ ), the boundary equation of the elliptic region,

$$
D_{x_{3}} u_{T\left\lceil x_{3}=0\right.}^{k}+\Xi_{T} u_{T\left\lceil x_{3}=0\right.}^{k} \underset{k \rightarrow+\infty}{\longrightarrow} 0 \text { in } \boldsymbol{L}_{\tilde{\rho}, \partial}^{2} .
$$

This implies in view of Lemma 4.6 a uniform Lopatinsky boundary equation on $\partial_{t} \varphi^{k}$, thus the propagation of the support of $\mu$, which is the same as that of $\mu_{L}$. The fact that $\eta^{\prime} \neq 0$ shows that $\tilde{\rho}$ cannot be hyperbolic for the longitudinal wave (in this case outgoing and incoming directions are not parallel, thus at least one is not parallel to $\vec{B}$ ). Consequently, $\tilde{\rho} \in \mathcal{G}_{L}$. More precisely, it is a diffractive point: the bicharacteristic passing through $\tilde{\rho}$ must stay parallel to $\vec{B}$, thus its direction is constant which is not possible for gliding rays because $\Omega$ has no contact of infinite order with its tangents. We are in the $\left(\mathcal{G}_{L}\right)$ case of Lemma 4.5.

- If $\tilde{\rho} \in \mathcal{G}_{T}$, then $\tilde{\rho} \in \mathcal{H}_{L}$. The fact that $\tilde{\rho} \in \mathcal{G}_{T}$ implies that $\tilde{\eta}^{\prime} \neq 0$, so directions $\tilde{\eta}_{L}^{+}$and $\tilde{\eta}_{L}^{-}$cannot be both parallel to $\vec{B}$. Consequently, the support of $\mu_{L}$ is a union of only incoming rays (or only outgoing rays). This gives a boundary equation of the following form:

$$
\partial_{x_{3}} \partial_{t} \varphi_{\left\lceil x_{3}=0\right.}^{k}+\iota \Lambda_{L} \partial_{t} \varphi_{\left\lceil x_{3}=0\right.}^{k} \underset{k \rightarrow+\infty}{\longrightarrow} 0 \text { in } \boldsymbol{L}_{\tilde{\rho}, \partial}^{2},
$$


where $\iota \in\{+1,-1\}$. Notice that $\partial_{t}$ is elliptic at $\tilde{\rho}$, so that we may rewrite this last property taking out all the $\partial_{t}$ and with $\boldsymbol{H}^{1}$ instead of $\boldsymbol{L}^{2}$. This yields, in view of Lemma 4.6, a uniform Lopatinsky boundary condition on $u_{T}^{k}$. The nullity of $\mu_{T}$ gives as before (by Prop. 3.18)

$$
u_{L}^{k}=-u_{T}^{k} \underset{k \rightarrow+\infty}{\longrightarrow} 0 \text { in } \boldsymbol{H}_{\tilde{\rho}}^{1}
$$

so that $\mu_{L}$ propagates, and in view of the particular form of its support, vanishes near $\tilde{\rho}$. This shows that this particular situation $\left(\tilde{\rho} \in \mathcal{G}_{T}\right.$ and $\left.\mu_{L} \neq 0\right)$ is impossible, and completes the proof of Lemma 4.5.

Definition 4.7. We shall call $\boldsymbol{B}$-admissible points the points of the boundary of $S_{b}^{*} M$ which are of one of the eight types described in Lemma 4.5.

\subsection{Conclusion of the proof}

Let $S:=\operatorname{supp} \mu_{L} \cup \operatorname{supp} \mu_{T}$, and BR the subset of $S_{b}^{*} M$, of all points $\rho$ satisfying one of the following properties:

- $x_{3}>0, \rho \in S \widehat{Z}_{L}$ and $\eta / / \vec{B}$

- $x_{3}>0, \rho \in S \widehat{Z}_{T}$ and $\eta \perp \vec{B}$;

- $x_{3}=0$ and $\rho$ is $\boldsymbol{B}$-admissible.

Let $\Phi_{T}(\rho, s)$ and $\Phi_{L}(\rho, s)$ be the bicharacteristic flows for the transversal and longitudinal waves. We shall define a local continuous flow on BR, denoted by

$$
\Phi(\rho, s)=\left(\Phi_{x^{\prime}}, \Phi_{x_{3}}, \Phi_{\xi^{\prime}}, \Phi_{\xi_{3}}\right),
$$

in the following way:

- if $\Phi_{x_{3}}(\rho, s)>0$ and $\Phi(\rho, s) \in S \widehat{Z}_{T}$, or if $\Phi(\rho, s)$ is a $\boldsymbol{B}$-admissible boundary point of the form (1) of Lemma 4.5, $\Phi$ is near $(\rho, s)$ the restriction to BR of the transversal bicharacteristic flow;

- if $\Phi_{x_{3}}(\rho, s)>0$ and $\Phi(\rho, s) \in S \widehat{Z}_{L}$, or if $\Phi(\rho, s)$ is a $\boldsymbol{B}$-admissible boundary point of the form (2) of Lemma 4.5, $\Phi$ is near $(\rho, s)$ the restriction to BR of the longitudinal bicharacteristic flow;

- if $\Phi(\rho, s)$ is $\boldsymbol{B}$-admissible of type $(T \rightarrow L)$, then

$$
\begin{aligned}
& \Phi(\rho, r)=\Phi_{T}(\rho, r), \text { if } r<s \\
& \Phi(\rho, r)=\Phi_{L}(\rho, r), \text { if } r>s ;
\end{aligned}
$$

- if $\Phi(\rho, s)$ is $\boldsymbol{B}$-admissible of type $(L \rightarrow T)$, then

$$
\begin{aligned}
& \Phi(\rho, r)=\Phi_{L}(\rho, r), \text { if } r<s \\
& \Phi(\rho, r)=\Phi_{T}(\rho, r), \text { if } r>s .
\end{aligned}
$$

In view of Lemma 4.3, $S$ (which a subset of BR) is stable under the flow $\Phi$ on $(0, T)$. Furthermore, if for some $\rho \in \mathrm{BR}$,

$$
\Phi(\rho, s) \underset{s \rightarrow \tilde{s}}{\longrightarrow} \tilde{\rho} \notin \mathrm{BR}, \tilde{s} \in(0, T),
$$


(thus $\tilde{\rho}$ is a boundary point which is not $\boldsymbol{B}$-admissible), then $\rho$ is not in $S$. Consequently, $S$ is a union of $\boldsymbol{B}$-resistant rays of life-length $T$. The assumption of non-existence of such rays made in Proposition 4.1 shows that $S$ is empty, which completes the proof.

\section{NECESSARY CONDITION}

Proposition 5.1. Assume that for all $T>0$, there exists a $\boldsymbol{B}$-resistant ray of life-length $T$. Then for all $T>0$, there exists a sequence $\left(u^{k}\right)$ of solutions of the Lamé system such that

$$
\begin{array}{r}
\left\|\partial_{t} u_{\uparrow t=0}^{k}\right\|_{\boldsymbol{L}^{2}}^{2}+\left\|u_{\uparrow t=0}^{k}\right\|_{\boldsymbol{H}_{0}^{1}}^{2} \underset{k \rightarrow+\infty}{\longrightarrow} 1 \\
\left\|u^{k} \wedge \boldsymbol{B}\right\|_{\boldsymbol{H}^{1}((0, T) \times \Omega)} \underset{k \rightarrow+\infty}{\longrightarrow} 0
\end{array}
$$

Corollary 5.2. Under the assumption of Proposition 5.1, the energy of the solutions of the magnetoelasticity equations does not decay uniformly. In other terms, the necessary condition of Theorem 1.2 holds.

Corollary 5.2 is a direct consequence of point b) of Proposition 2.5.

Proof of Proposition 5.1. This proof is inspired by the one of Theorem 4 of [3]. Denote by $Z$ one of the indices $T$ or $L$ and set $P_{Z}:=\Delta-\nu_{Z}^{2} \partial_{t}^{2}$. We start by an elementary remark:

Remark 5.3. Let $E$ be a vector subspace of $\mathbb{C}^{3}$ and $\pi_{E}$ the orthogonal projection on $E$. The defect measure of $\pi_{E} u_{Z}^{k}$ is $\pi_{E}^{*} \mu_{Z} \pi_{E}$. Furthermore, $P_{Z} \pi_{E} u_{Z}^{k}=0$, so that the theorem of propagation 3.12 holds for the measure $\pi_{E}^{*} \mu_{Z} \pi_{E}$ if a uniform Lopatinsky boundary condition holds on $\pi_{E} u_{Z}^{k}$. Notice that any scalar uniform Lopatinsky condition on $u_{Z}^{k}$ yields such a condition on $\pi_{E} u_{Z}^{k}$. If $\pi_{E}^{*} \mu_{Z} \pi_{E}=\mu_{Z}$, the measure $\mu_{Z}$ will be said to be polarized along $E$. If $E$ is the line generated by a vector $H$ of $\mathbb{C}^{3}$ we shall also use the phrase "polarized along $H$ ". If both measures $\mu_{T} \mathbb{1}_{]-\varepsilon, T+\varepsilon[}$ and $\mu_{L} \mathbb{1}_{]-\varepsilon, T+\varepsilon[}$ are polarized along $\boldsymbol{B}$, then condition (5.2) is fulfilled.

Let $T^{\prime}>T$. Consider a $\boldsymbol{B}$-resistant ray defined on an open interval $I$ of length $T^{\prime}$ :

$$
\gamma(s)=\left(t_{\gamma}(s), y_{\gamma}(s), \tau_{\gamma}(s), \eta_{\gamma}(s)\right)=\left(x_{\gamma}(s), \xi_{\gamma}(s)\right)
$$

If $T^{\prime}$ is large enough, then one of the two following assertions holds:

a) $\gamma(I)$ contains an interior point;

b) $y_{\gamma}(I)=\Gamma \subset \partial \Omega$ where $\Gamma$ is a closed curved, contained in a plane $P$ which is normal to $\boldsymbol{B}$, boundary of a convex subset of $P$, and such that on $\Gamma, \mathbf{n}$ is orthogonal to $\boldsymbol{B}$.

Case b) occurs when there exists an infinite boundary $\boldsymbol{B}$-resistant ray. This case reduces to case a), choosing a transversal ray contained in $P$ which only meets the boundary at hyperbolic points.

Thus, we may assume that $\gamma(I)$ has an interior point. We may also assume, possibly translating the origin, that this interior point is $\gamma(0)$, and that $\left(t_{\gamma}(0), y_{\gamma}(0)\right)=(0,0)$. Recall that the magnetic field is vertical: $\boldsymbol{B}=(B, 0,0)$. We shall denote by $-T^{-}$and $T^{+}$the extremal points of $I: I=\left(-T^{-}, T^{+}\right)$.

If $\eta_{\gamma}(0)$ is parallel to $\boldsymbol{B}$ (i.e. if $\gamma(0)$ is in the longitudinal characteristic set), choose a non-zero function $\varphi \in C_{0}^{\infty}(\Omega)$, and set

$$
\varphi^{k}(y)=K^{-1} k^{-5 / 4} e^{i k y_{1}} \varphi(\sqrt{k} y), \quad u_{0}^{k}=\nabla \varphi^{k}, \quad u_{1}^{k}=i k c_{L} u_{0}^{k} .
$$

Where $u^{k}$ is the solution of the Lamé system with initial data $\left(u^{k}, \partial_{t} u^{k}\right)_{\lceil t=0}=\left(u_{0}^{k}, u_{1}^{k}\right)$. Then

$$
\left\|u_{0}^{k}\right\|_{H^{1}} \underset{k \rightarrow+\infty}{\longrightarrow} K^{-1}\|\varphi\|_{L^{2}}, \quad\left\|u_{1}^{k}\right\|_{L^{2}} \underset{k \rightarrow+\infty}{\longrightarrow} K^{-1}\|\varphi\|_{L^{2}} .
$$


Thus, condition (5.1) is fulfilled with an appropriate choice of $K$.

For small $t$, by finite speed of propagation for the wave equation, $u_{L}^{k}$ has compact support in $\Omega$. Thus $u_{T}^{k}=0$ and $u_{L}^{k}=u^{k}$. As a consequence, for small $t$,

1. $\mu_{T}=0$

2. $\mu_{L}$ is polarized along $\boldsymbol{B}$;

3. the projection of the support of $\mu_{L}$ on $\mathbb{R}_{t} \times \bar{\Omega}$ is contained in $x_{\gamma}(I)$.

If $\eta_{\gamma}(0)$ is orthogonal to $\boldsymbol{B}$, we construct a sequence of solution of the Lamé system, with the following initial data $(c f .[3])$ :

$$
\psi^{k}=K^{-1} k^{-5 / 4} e^{i k y_{1}} \psi(\sqrt{k} y), \quad u_{0}^{k}=\operatorname{curl}\left(0,0,-\psi^{k}\right), \quad u_{1}^{k}=i k c_{T} u_{0}^{k}
$$

In this case, condition (5.1) is fullfilled for an appropriate $K$ and the defect measures satisfy the following properties for small $t$ :

1. $\mu_{L}=0$;

2. $\mu_{T}$ is polarized along $\boldsymbol{B}$;

3. the projection of the support of $\mu_{T}$ on $\mathbb{R}_{t} \times \bar{\Omega}$ is contained in $x_{\gamma}(I)$.

To show (5.2), we shall prove that both measures $\mu_{T}$ and $\mu_{L}$, are, for $t \in I$, polarized along $\boldsymbol{B}$. For $t>0$, we shall denote by $\mathcal{P}(t)$ the following property: in a neighbourhood of $[0, t]$, both measures $\mu_{L}$ and $\mu_{T}$ are polarized along $B$ and the projections of their support on $\mathbb{R}_{t} \times \bar{\Omega}$ are contained in $x_{\gamma}(I)$.

Let $\mathcal{T}$ be the set of $t$ in $\left[0, T^{+}\right)$such that $\mathcal{P}(t)$ holds. By its definition, $\mathcal{T}$ is an open subset of $\left[0, T^{+}\right)$. We have just shown that 0 is in $\mathcal{T}$. We shall now prove that $\mathcal{T}$ is closed. We will use the following lemma:

Lemma 5.4. Let $\tilde{\rho}=(\tilde{t}, \tilde{y}, \tilde{\tau}, \tilde{\eta}) \in S_{b}^{*} M$. If $\mu_{T} \mathbb{1}_{t<\tilde{t}}$ and $\mu_{L} \mathbb{1}_{t<\tilde{t}}$ vanish in a neighbourhood of $\tilde{\rho}$, the measures $\mu_{T}$ and $\mu_{L}$ also vanish in a neighbourhood of $\tilde{\rho}$.

This is a trivial assertion in the interior of $\Omega$ by the propagation of both measures. Near a point of the boundary of $\Omega$, one may show Lemma 5.4 using the Dirichlet boundary condition on $u^{k}$ and the theorem of propagation 3.12, together with the same type of arguments as in Lemma 4.5.

Let $s_{0}>0$ such that $\mathcal{P}\left(s_{0}\right)$ holds for $s<s_{0}$. We must check that $\mathcal{P}\left(s_{0}\right)$ holds. Five cases arise, depending on the nature of $\rho:=\gamma\left(s_{0}\right)$.

i) $\rho$ is an interior point.

$\mathcal{P}\left(s_{0}\right)$ is obvious in view of the propagation of both measures in the interior of $\Omega$.

ii) $\rho$ is of the type (1) of Lemma 4.5 .

This case, where $\mu_{L}$ vanishes for time $t<t_{\gamma}\left(s_{0}\right)$ near $t_{\gamma}\left(s_{0}\right)$, was studied in [3]. The authors show that $\mu_{L}$ remains null for times greater than $t_{\gamma}\left(s_{0}\right)$ and that $\mu_{T}$ propagates near $\gamma\left(s_{0}\right)$, in such a way that in our case, its polarization along $\boldsymbol{B}$ is preserved. In particular property $\mathcal{P}\left(s_{0}\right)$ holds. The next three cases are represented in Figure 3.

iii) $\rho$ is of the type $\left(\mathcal{H}_{L}\right)$ of Lemma $4.5: y_{\gamma}^{\prime}\left(s_{0}\right) \in \partial \Omega, \eta_{\gamma}^{\prime}\left(s_{0}\right)=0$ and $\mathbf{n} / / \boldsymbol{B}$.

In view of Lemma 5.4, the support of the measure $\mu_{L}$ is contained, near $\rho$ in the union of the longitudinal ray coming in $\rho$ and the longitudinal ray going out of $\rho$. The support of $\mu_{T}$, if not empty, if the transversal ray going out of $\rho$. Let $E$ be the plane orthogonal to $\boldsymbol{B}$ in $\mathbb{C}^{3}$. The polarization of $\mu_{L}$ along $\boldsymbol{B}$ shows that $\pi_{E}^{*} \mu_{L} \pi_{E}=0$ and thus, by Remark 5.3 and the standard hyperbolic theory of Proposition 3.14,

$$
\pi_{E} u_{T\left\lceil x_{3}=0\right.}^{k}=-\pi_{E} u_{L\left\lceil x_{3}=0\right.}^{k} \underset{k \rightarrow \infty}{\longrightarrow} 0 \text { in } \boldsymbol{H}_{\rho, \partial}^{1}
$$

which implies, using again Remark 5.3 that $\pi_{E}^{*} \mu_{T} \pi_{E}$ propagates along the transversal flow near $\gamma\left(s_{0}\right)$. Thus $\pi_{E}^{*} \mu_{T} \pi_{E}$ vanishes near $\rho$. But $\mu_{T}$ is polarized orthogonally to its direction of propagation which is exactly $\boldsymbol{B}$ on the support of $\mu_{T}$ near $\rho$. This show that $\mu_{T}$ vanishes near $\rho$, completing the proof of $\mathcal{P}\left(s_{0}\right)$. 
iv) $\rho$ is of the type $\left(\mathcal{G}_{L}\right)$ of Lemma $4.5: \rho$ is a diffractive point for the longitudinal wave, and $\eta_{\gamma}^{\prime}\left(s_{0}\right)$ is parallel to $\boldsymbol{B}$.

Then $\rho \in \mathcal{H}_{T} \cup \mathcal{E}_{T}$. Furthermore, if $\rho \in \mathcal{H}_{T}, \mu_{T} \mathbb{1}_{t<t_{\gamma}\left(s_{0}\right)}$ vanishes near $\rho$. Thus, according to standard elliptic or hyperbolic theory (cf. Props. 3.15 and 3.16), $u_{T}^{k}$ satisfies a boundary condition of the following form:

$$
D_{x_{3}} u_{T\left\lceil x_{3}=0\right.}^{k}=A u_{T\left\lceil x_{3}=0\right.}^{k}+o(1) \text { in } \boldsymbol{L}_{\rho, \partial}^{2}, \quad A \in \mathcal{A}_{\partial}^{1},
$$

where $\sigma_{1}(A)=-i \sqrt{\left\|\eta^{\prime}\right\|^{2}-\nu_{T}^{2} \tau^{2}}$ in the elliptic case and $\sigma_{1}(A)=-\sqrt{\nu_{T}^{2} \tau^{2}-\left\|\eta^{\prime}\right\|^{2}}$ in the hyperbolic case. This yields, in view of Lemma 4.6, an uniform Lopatinsky boundary condition on $\varphi^{k}$ :

$$
\varphi_{\left\lceil x_{3}=0\right.}^{k}=B_{-1} D_{x_{3}} \varphi_{\left\lceil x_{3}=0\right.}^{k}+o(1) \text { in } \boldsymbol{H}_{\rho, \partial}^{1} .
$$

As a consequence, the support of $\mu$ (and that of $\mu_{L}$ ) propagates near $\rho$. The polarization of $\mu_{L}$ along $\boldsymbol{B}$ is immediate. The nullity of $\mu_{T}$ near $\rho$ remains to be checked. This is a general property in the elliptic case $\rho \in \mathcal{E}_{T}$. In the hyperbolic case, first note that the propagation theorem of Burq and Lebeau ([3], Thm. 1) implies with the boundary condition (5.3) that $\mu$ is invariant by the longitudinal flow near diffractive points. So the total mass of $\mu_{L}$ is preserved by time, for $t$ close enough to $t_{\gamma}\left(s_{0}\right)$. The next lemma, which is a measure version of the conservation of energy for the Lamé system, completes the proof of $\mathcal{P}\left(s_{0}\right)$ :

Lemma 5.5. Let $\varphi \in C_{0}^{\infty}(\mathbb{R})$. Then

$$
\left\langle\mu_{T}+\mu_{L}, \varphi^{\prime}(t)\right\rangle=0
$$

Thus the total mass of the measure $\left(\mu_{T}+\mu_{L}\right)_{\mid t=s}$ is well defined, and does not depend on $s$.

Proof. In this proof, we denote by $x=(t, z) \in \mathbb{R} \times \Omega$ the global space-time variable. By the equation $\left(\partial_{t}^{2}-\right.$ $\left.\Delta_{e}\right) u^{k}=0$,

$$
\operatorname{Re} \int \partial_{t}^{2} u^{k} \partial_{t} \bar{u}^{k} \varphi(t) \mathrm{d} x-\operatorname{Re} \int \Delta_{e} u^{k} \partial_{t} \bar{u}^{k} \varphi(t) \mathrm{d} x=0
$$

Set $\nabla_{e} u:=(\varsigma \nabla u,(\lambda+\varsigma) \operatorname{div} u) \in \mathbb{C}^{4}$. A simple integration by parts yields

$$
\int \varphi^{\prime}(t)\left|\partial_{t} u^{k}\right|^{2} \mathrm{~d} x+\int \varphi^{\prime}(t)\left|\nabla_{e} u^{k}\right|^{2} \mathrm{~d} x=0
$$

Using another integration by parts, and then the decoupling Lemma 3.28,

$$
\begin{gathered}
-\int \partial_{t}^{2} u^{k} \bar{u}^{k} \varphi^{\prime}(t) \mathrm{d} x-\int \Delta_{e} u^{k} \bar{u}^{k} \varphi^{\prime}(t) \mathrm{d} x=o(1) \text { as } k \rightarrow+\infty \\
\int\left(\partial_{t}^{2}+c_{T}^{2} \Delta\right) u_{T}^{k} \bar{u}_{T}^{k} \varphi^{\prime}(t) \mathrm{d} x+\int\left(\partial_{t}^{2}+c_{L}^{2} \Delta\right) u_{L}^{k} \bar{u}_{L}^{k} \varphi^{\prime}(t) \mathrm{d} x=o(1) \text { as } k \rightarrow+\infty,
\end{gathered}
$$

and thus, by the definition of $\mu_{T}$ and $\mu_{L}$,

$$
\left\langle\mu_{T}, \frac{\tau^{2}+c_{T}^{2}\|\eta\|^{2}}{2 \tau^{2}} \varphi^{\prime}(t)\right\rangle+\left\langle\mu_{L}, \frac{\tau^{2}+c_{L}^{2}\|\eta\|^{2}}{2 \tau^{2}} \varphi^{\prime}(t)\right\rangle=0
$$

This completes the proof, noting that on the support of $\mu_{T}$ (respectively $\left.\mu_{L}\right), c_{T}\|\eta\|\left(\right.$ respectively $\left.c_{L}\|\eta\|\right)$ is equal to $\tau$. 


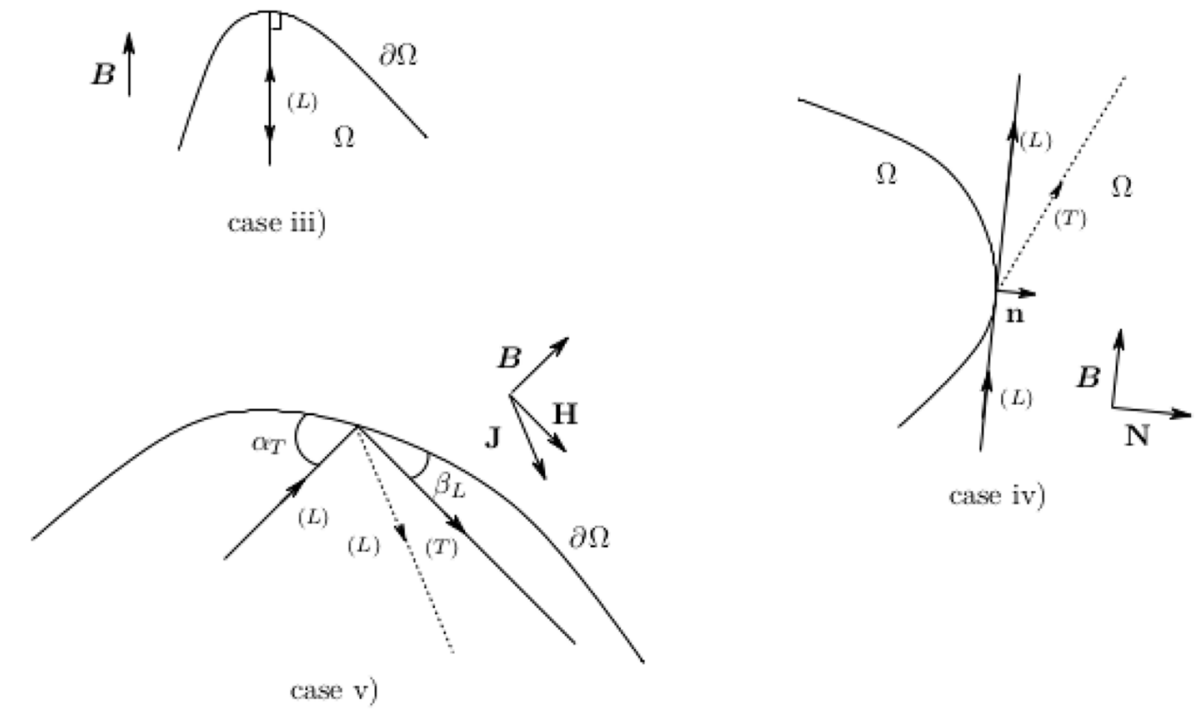

Figure 3. Cases iii), iv) and v).

Lemma 5.5 and the mass conservation of $\mu_{L}$ imply that the mass of $\mu_{T}$ is also preserved near $\rho$, which shows that $\mu_{T}$ vanishes in a neighbourhood of $\rho$.

v) $\rho$ is of the type $(L \rightarrow T)$ of Lemma 4.5.

In view of Lemma 5.4 and of the assumption $P(s)$ for $s<s_{0}$, the support of $\mu_{L}$ is contained in the two longitudinal half-rays passing through $\rho$, and that of $\mu_{T}$ is only contained in the ray going out of $\rho$. To prove $P\left(s_{0}\right)$, it remains to show that $\mu_{L}=0$ along the longitudinal ray going out of $\rho$. We shall do so by a simple polarization argument. Let $H$ (respectively $J$ ) be a unitary vector of $\mathbb{C}^{3}$ parallel to the direction of the transversal (respectively longitudinal) ray going out of $\rho$. The polarization of $\mu_{T}$ shows that $\pi_{H}^{*} \mu_{T} \pi_{H}$ is null, so that $\pi_{H}^{*} \mu_{L} \pi_{H}$ propagates near $\rho$. Now, $H$ is orthogonal to the direction of the longitudinal ray coming in $\rho$, so that $\pi_{H}^{*} \mu_{L} \pi_{H}$ vanishes, along incoming rays but also, in view of the propagation, along outgoing rays. Furthermore $\mu_{L} \mathbb{1}_{t>t_{\gamma}}\left(s_{0}\right)$ is polarized along $J$. It is easy to see that this last measure vanishes. Indeed, the polarization of $\mu_{L}$ implies

$$
\mathbb{1}_{t>t_{\gamma}\left(s_{0}\right)} \pi_{J}^{*} \pi_{H}^{*} \pi_{J}^{*} \mu_{L} \pi_{J} \pi_{H} \pi_{J}=\mathbb{1}_{t>t_{\gamma}\left(s_{0}\right)} \pi_{J}^{*} \pi_{H}^{*} \mu_{L} \pi_{H} \pi_{J}=0
$$

But

$$
\pi_{J} \pi_{H} \pi_{J}=\frac{<H, J>^{2}}{|H|^{2}|J|^{2}} \pi_{J}
$$

Noting that $H$ and $J$ are not orthogonal this yields the nullity of $\mu_{L} \mathbb{1}_{t>t_{\gamma}\left(s_{0}\right)}$ in a neighbourhood of $\rho$. vi) $\rho$ is of the type $(T \rightarrow L)$ of Lemma 4.5.

One may argue as before, showing that for every vector $K$ orthogonal to $J, \pi_{K}^{*} \mu_{T} \pi_{K}=0$ near $\rho$, which implies the nullity of $\mu_{T} \mathbb{1}_{t>t_{\gamma\left(s_{0}\right)}}$ near $\rho$.

The proof is completed by reversing time, which yields $\mathcal{P}(s)$ for $-T^{-}<s<0$.

\section{Appendix A. Regularity of Boundary value PRoblems}

The following proposition concerns solutions of a partial differential equation which is transverse to the boundary of an open set. It says that for such functions, the control of derivatives which are tangential to the boundary suffices to control all the derivatives. As in Section 3, we shall work in an open subset $\bar{X}$ of $\overline{\mathbb{R}}_{+}^{n+1}$, of 
the form $X^{\prime} \times\left[0, l\left[\right.\right.$, where $X^{\prime}$ is an open subset of $\mathbb{R}^{n}$. Let $P$ be a differential operator of degree $r$ on $\bar{X}$, of the following form:

$$
P=\sum_{j=0 . . r} Q_{r-j} \partial_{x_{n}}^{j}
$$

where the $Q_{j}$ 's are $N \times N$ matrices of tangential differential operators, with $C^{\infty}(\bar{X})$ coefficients, and $Q_{0}$ is the identity of $\mathbb{C}^{N}$. To simplify the following statements, we suppose $u \in C^{\infty}(\bar{X})$.

Proposition A.1. Let $s \geq 0, j \in \mathbb{N}$, and suppose that $P u=0$ on $\bar{X}$. Then

$$
\forall \varphi \in C_{0}^{\infty}(\bar{X}), \quad \exists \tilde{\varphi} \in C_{0}^{\infty}(\bar{X}), \quad\left\|\varphi \partial_{x_{n}}^{j} u\right\|_{\boldsymbol{H}^{s-j}} \leq C\|\tilde{\varphi} u\|_{L^{2}\left(0, l ; \boldsymbol{H}^{s}\left(X^{\prime}\right)\right)},
$$

where $C$ does not depend on $u$. Likewise (assuming again $P u=0$ ),

$$
\forall \varphi \in C_{0}^{\infty}(\bar{X}), \quad \exists \tilde{\varphi} \in C_{0}^{\infty}(\bar{X}), \quad\|\varphi u\|_{L^{2}\left(0, l ; \boldsymbol{H}^{-s}\left(X^{\prime}\right)\right)} \leq C\|\tilde{\varphi} u\|_{\boldsymbol{H}^{-s}(X)} .
$$

\section{Appendix B. Proof of Proposition 3.18}

As mentioned in the introduction of Section 3, whe shall assume that each $u^{k}$ is smooth enough, so that all the quantities appearing in the following calculation are well defined and finite. Let

$$
A=A_{0} D_{x_{n}}, A_{0} \in \mathcal{A}^{0}, \mathcal{C}^{k}:=\left([P, A] u^{k}, u^{k}\right) .
$$

Take the support of $A_{0}$ in a small enough neighbourhood of $\tilde{\rho}$. The operator $P$ is formally self-adjoint. A simple integration by parts yields

$$
\mathcal{C}^{k}=\underbrace{-\left(A P u^{k}, u^{k}\right)+\left(A u^{k}, P u^{k}\right)}_{0}-\left(A u_{\uparrow x_{n}=0}^{k}, i D_{x_{n}} u_{\uparrow x_{n}=0}^{k}\right)_{\partial}+\left(i D_{x_{n}} A u_{\uparrow x_{n}=0}^{k}, u_{\uparrow x_{n}=0}^{k}\right)_{\partial},
$$

where $(., .)_{\partial}$ is the $\boldsymbol{L}^{2}$ scalar product on $\left\{x_{n}=0\right\}$, with respect to the measure $\sqrt{g_{\left\lceil x_{n}=0\right.}} \mathrm{d} x^{\prime} \mathrm{d} t$. We have

$$
\begin{aligned}
D_{x_{n}} A_{0} D_{x_{n}} u^{k} & =A_{0} D_{x_{n}}^{2} u^{k}+\left[D_{x_{n}}, A_{0}\right] D_{x_{n}} u^{k}=-A_{0} Q u^{k}+R_{0} D_{x_{n}} u^{k}, \quad R_{0} \in \mathcal{A}^{0} \\
\mathcal{C}^{k} & =i\left(A_{0} D_{x_{n}} u^{k}, D_{x_{n}} u^{k}\right)_{\partial}-i\left(A_{0} Q u^{k}, u^{k}\right)_{\partial}+\left(R_{0} D_{x_{n}} u^{k}, u^{k}\right)_{\partial} .
\end{aligned}
$$

So, using condition (3.12),

$$
\begin{array}{r}
\mathcal{C}^{k}=i\left(A_{0} D_{x_{n}} u^{k}, D_{x_{n}} u^{k}\right)_{\partial}-i\left(A_{0} Q B_{-1} D_{x_{n}} u^{k}, B_{-1} D_{x_{n}} u^{k}\right)_{\partial}-i\left(A_{0} Q h^{k}, B_{-1} D_{x_{n}} u^{k}\right)_{\partial} \\
-i\left(A_{0} Q B_{-1} D_{x_{n}} u^{k}, h^{k}\right)_{\partial}+\left(R_{0} D_{x_{n}} u^{k}, B_{-1} D_{x_{n}} u^{k}\right)_{\partial}+o(1), k \rightarrow+\infty
\end{array}
$$

Take $A_{0}$ of the form $T_{0}^{*} T_{0}$, where $T_{0} \in \mathcal{A}_{0}$ is scalar, elliptic at $\tilde{\rho}$ and has support in a small neighbourhood of $\tilde{\rho}$. Then

$$
\begin{gathered}
\mathcal{C}^{k}=i\left(E_{0} D_{x_{n}} u^{k}, D_{x_{n}} u^{k}\right)_{\partial}-i\left(T_{0} Q h^{k}, B_{-1} T_{0} D_{x_{n}} u^{k}\right)-i\left(Q B_{-1} T_{0} D_{x_{n}} u^{k}, T_{0} h^{k}\right)+o(1), k \rightarrow+\infty \\
E_{0} \in \mathcal{A}^{0}, E_{0}=T_{0}^{*} T_{0}-B_{-1}^{*} T_{0}^{*} T_{0} Q B_{-1}+B_{-1}^{*} R_{0} .
\end{gathered}
$$

Denoting by $t_{0}$ the principal symbol (which is scalar) of $T_{0}$, we have

$$
\sigma_{0}\left(E_{0}\right)=\left|t_{0}\right|^{2}\left(1-b_{-1}^{*} b_{-1} q_{2}\right) .
$$


Since $q_{2}$ vanishes at $\tilde{\rho}$, we may choose $t_{0}$ with support in a small enough neighbourhood of $\tilde{\rho}$ such that

$$
\sigma\left(E_{0}\right) \geq 1 / 2\left|t_{0}\right|^{2}
$$

(in the sense of quadratic positive Hermitian forms). The weak Gårding inequality, applied to the operator $E_{0}-1 / 2 T_{0}^{*} T_{0}$, thus yields

$$
\liminf _{k \rightarrow+\infty} \operatorname{Re}\left(E_{0} D_{x_{n}} u^{k}, D_{x_{n}} u^{k}\right)-\frac{1}{2}\left\|T_{0} D_{x_{n}} u^{k}\right\|_{L_{\partial}^{2}}^{2} \geq 0
$$

This implies, using the convergence to 0 of $h^{k}$ in $\boldsymbol{H}_{\tilde{\rho}}^{1}$

$$
\liminf _{k \rightarrow+\infty} \operatorname{Im} \mathcal{C}^{k} \geq \frac{1}{4}\left\|T_{0} D_{x_{n}} u^{k}\right\|_{\boldsymbol{L}_{\partial}^{2}}^{2}
$$

Thus $D_{x_{n}} u_{\uparrow x_{n}=0}^{k}$ is bounded in $\boldsymbol{L}_{\tilde{\boldsymbol{\rho}}, \partial}^{2}$, which yields, with the boundary condition, that $u_{\left\lceil x_{n}=0\right.}^{k}$ is bounded in $\boldsymbol{H}_{\tilde{\rho}, \partial}^{1}$. The proof of (3.17) is complete.

When $\mu$ is null near $\tilde{\rho}$, we have

$$
\lim _{k \rightarrow+\infty} \mathcal{C}^{k}=\left\langle\mu, \frac{\left\{p, a_{1} \xi_{n}\right\}}{\tau^{2}}\right\rangle=0
$$

which yields (3.18).

Point b) of Proposition 3.18 may be seen as a consequence of the propagation theorem of [3]. The assumptions (3.19) imply that any uniform Lopatinsky boundary condition holds on the traces of $u^{k}$, which shows that the measure $\mu$ propagates near $\tilde{\rho}$ with any smooth multiplicative factor, which is impossible unless $\mu$ is null.

\section{REFERENCES}

[1] E. Andreou and G. Dassios, Dissipation of energy for magnetoelastic wavec in a conductive medium. Quart. Appl. Math. 55 (1997) 23-39.

[2] C. Bardos, G. Lebeau and J. Rauch, Sharp sufficient conditions for the observation, control and stabilization of waves from the boundary. SIAM J. Control Optim. 305 (1992) 1024-1065.

[3] N. Burq and G. Lebeau, Mesures de défaut de compacité, application au système de Lamé. Ann. Sci. de l'École Normale Supérieure (4) 34 (2001) 817-870. Mesures de défaut de compacité, application au système de Lamé. A paraître aux Annales de L'Ecole Normale Supérieure (1999).

[4] N. Burq and P. Gérard, Condition nécessaire et suffisante pour la contrôlabilité exacte des ondes. (French). Comptes Rendus de l'Académie des Sciences Paris Sér. I Math. 325 (1997) 749-752.

[5] R.C. Charão, J.C. Oliveira and G. Perla Menzala, Energy decay rates of magnetoelastic waves in a bounded conductive medium. Discr. Continu. Dyn. Syst. 25 (2009) 797-821.

[6] R.C. Charão, J.C. Oliveira and G. Perla Menzala, Decay rates of magnetoelastic waves in an unbounded conductive medium. Electr. J. Differ. Equ. 2011 (2011) paper no. 127, 14 p.

[7] C.R. da Luz and J.C. Oliveira, Asymptotic behavior of solutions for the magneto-thermo-elastic system in $\mathbb{R}^{3}$. J. Math. Anal. Appl. 432 (2015) 1200-1215.

[8] M. de Lima Santos and J.E. Muñoz Rivera, Polynomial stability to three dimensional magnetoelasticity. Acta Appl. Math. 76 (2003) 265-281.

[9] T. Duyckaerts, Stabilization of the linear system of magnetoelasticity. Preprint arXiv:0407257 (2004).

[10] P. Gérard, Microlocal defect measures. Commun. Partial Differ. Equ. 16 (1991) 1761-1794.

[11] L. Hörmander, The analysis of linear partial differential operators. III. Vol. 274 of Grundlehren der Mathematischen Wissenschaften [Fundamental Principles of Mathematical Sciences]. Springer-Verlag, Berlin (1994).

[12] G. Lebeau, Équation des ondes amorties. In Algebraic and geometric methods in mathematical physics (Kaciveli, 1993), vol. 19 of Math. Phys. Stud. Kluwer Acad. Publ., Dordrecht (1996) 73-109.

[13] G. Lebeau and L. Robbiano, Stabilisation de l'équation des ondes par le bord. Duke Math. J. 86 (1997) 465-491.

[14] G. Lebeau and E. Zuazua, Null-controllability of a system of linear thermoelasticity. Arch. Ratl. Mech. Anal. 141 (1998) $297-329$. 
[15] G. Lebeau and E. Zuazua, Decay rates for the three-dimensional linear system of thermoelasticity. Arch. Ratl. Mech. Anal. 148 (1999) 179-231.

[16] T.F. Ma, J.M. Rivera, H.P. Oquendo and F.M.S. Suárez, Polynomial stabilization of magnetoelastic plates. IMA J. Appl. Math. 79 (2014) 241-253.

[17] R. Melrose and J. Sjöstrand, Singularities of boundary value problems I. Commun. Pure Appl. Math. 16 (1991) $1761-1794$.

[18] J.E. Muñoz Rivera and R. Racke, Polynomial stability in two-dimensional magnetoelasticity. IMA J. Appl. Math. 66 (2001) $269-283$.

[19] J.E. Muñoz Rivera and R. Racke, Magneto-thermo-elasticity. Large-time behavior for linear systems. Adv. Differ. Equ. 6 (2001) 359-384.

[20] G. Perla Menzala and E. Zuazua, Energy decay of magnetoelastic waves in a bounded conductive medium. Asymp. Anal. 18 (1998) 349-362.

[21] R. Quintanilla and R. Racke, Stability in thermoelasticity of type III. Discr. Continu. Dyn. Syst. Ser. B 3 (2003) 383-400.

[22] L. Tartar, H-measures, a new approach for studying homogenisation, oscillations and concentration effects in partial differential equations. Proc. Roy. Soc. Edinbur. 115-A (1990) 193-230.

[23] R. Temam, Navier-Stokes equations. Studies in mathematics and its applications, North-Holland 1979.

[24] X. Zhang, and E. Zuazua, Decay of solutions of the system of thermoelasticity of type III. Commun. Contemp. Math. 5 (2003) $25-83$. 